\title{
Detection of a structural impact in liquid metal flow during test runs of the EURISOL target mock-up
}

\author{
Rade Ž. Milenkovićc $^{\mathrm{a}, *}$, Karel Samec ${ }^{\mathrm{a}}$, Sergejs Dementjevs ${ }^{\mathrm{a}}$, Alexej Flerov ${ }^{\mathrm{b}}$, Werner Wagner ${ }^{\mathrm{a}}$ \\ a Paul Scherrer Institut, $\mathrm{CH}-5232$ Villigen PSI, Switzerland \\ ${ }^{\mathrm{b}}$ Institute of Physics of the University of Latvia, LV-2156 Salaspils, Latvia
}

\section{A R T I C L E I N F O}

\section{Article history:}

Received 10 July 2009

Accepted 12 July 2009

\section{Keywords:}

EURISOL

MEGAPIE

Spallation target

Liquid metal

CFD

Structural vibrations

Resonance

Cavitation

Time-frequency analysis

\section{A B S T R A C T}

The many possible applications of Liquid Metal (LM) technology promise improved thermal efficiency, which can provide much-needed impetus to finding solutions for the growing world energy needs, as well as increasing the accuracy and power of certain delicate scientific apparatus used in the materials and biological sciences. The development of appropriate safeguards and instrumentation is critical to continued progress in this area of technology.

During the hydraulic test of the EURISOL liquid metal target, a component failure was registered by the instrumentation; its detection in the presence of liquid metal flow was critical to ensuring the safety of both the personnel and the facility during a difficult test. The methods by which the failure was recorded and analysed are the subject of the paper.

(c) 2009 Elsevier B.V. All rights reserved.

\section{Q3 1. Introduction}

Within the context of the 6th framework program for research development, the European Union has supported the EURISOL

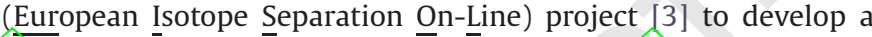
novel isotope production facility. In order to achieve high yields of rare isotopes it was necessary to increase the flux of the neutron source, which serves to generate isotopes by fissioning uranium carbide (UC) targets arranged circumferentially around it. This has led PSI to develop a very compact high-power neutron source based on liquid metal that emits neutrons when hit by a $4 \mathrm{MW}$ proton beam. The configuration chosen to solve the complex cooling requirements posed at the point of entry of the beam, the so-called beam window led PSI to study and test the feasibility of using thin flow vanes for accelerating whilst stabilizing the liquid metal flow on the window.

The manufacture and design of the flow vanes were a major challenge and their weakness resulted in a partial detachment of the flow vane structure, which impacted the hull. The instrumentation used to monitor the status of the target picked up the resulting signals, which are described fully in the current paper.

Two structural-hydraulic tests of the EURISOL neutron converter target mock-up, named METEX 1 [7] and METEX 2

\footnotetext{
* Corresponding author. Tel.: +41563104453.

E-mail address: rade.milenkovic@psi.ch (R.Ž. Milenković).
}

(MErcury Target EXperiments 1 and 2), have been conducted by PSI (Paul Scherrer Institut, Switzerland) in cooperation with IPUL (Institute of Physics of the University of Latvia, Latvia) and CERN (Conseil Européen pour la Recherche Nucléaire-European Organization for Nuclear Research).

During test runs of the EURISOL target mock-up for configuration with flow vanes, which were performed in December 2008, strain, acceleration and sound data were recorded at high sampling rates. These tests were carefully conducted, after having applied various safety precautions, since it was shown by Samec et al. [5] that the design of the weld between the supports and flow vanes was prone to high-cycle fatigue and by Milenkovic et al. [7] that the configuration with flow vanes had inherent weaknesses, which could lead to the failure of the vanes. The target mock-up has been designed and produced by the Paul Scherrer Institute (PSI); it draws significantly on recent experience obtained with the liquid metal target of Megawatt Pilot Experiment-MEGAPIE (Megawatt Pilot Experiment) for the SINQ neutron spallation source at PSI [10].

Phenomena related to flow-induced vibrations and flowexcited acoustic resonances are serious design issues encountered in many engineering applications, especially in nuclear industry [9]. Separated shear flows, flows through narrow gaps, annular flows, leakage flows and flows through restricted passages are very sensitive to changes in flow area. Thus, stiff or flexible structures embedded in such flows are very susceptible to a variety of flow-excited instabilities. doi:10.1016/j.nima.2009.07.019 
Fig. 1 shows a current design of the flow vanes. As already highlighted by Samec [4,5] and Milenkovic et al, [7], the flow vanes are incorporated into the design of the mock-up in order to direct and accelerate the flow to the surface of the beam entrance window (BEW). The large eddy simulation (LES) calculations (Fig. 2) showed that this aim was achieved, but some areas of concern existed with regard to the flow-induced vibrations as summarized below:

- The flow-induced vibrations, generated by the liquid metal

\section{a}

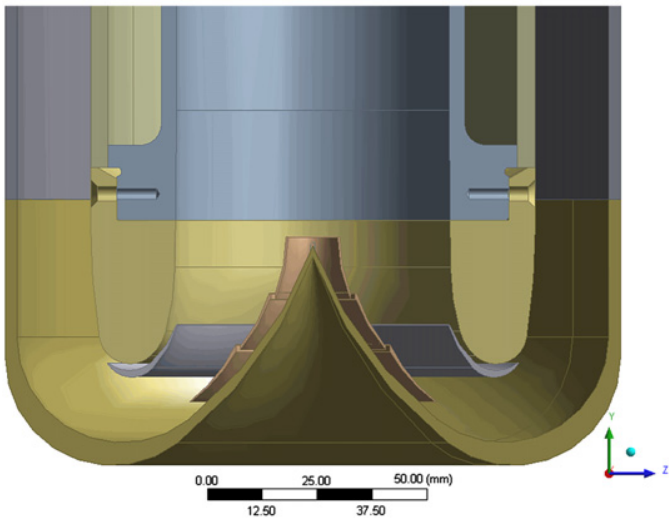

b
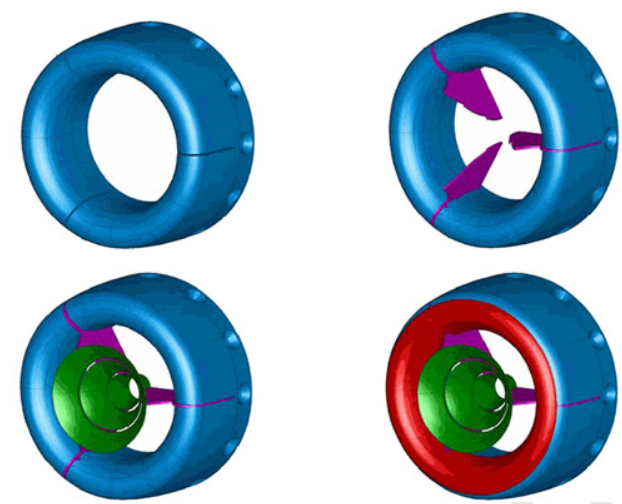

Fig. 1. Design of the flow vanes.

a

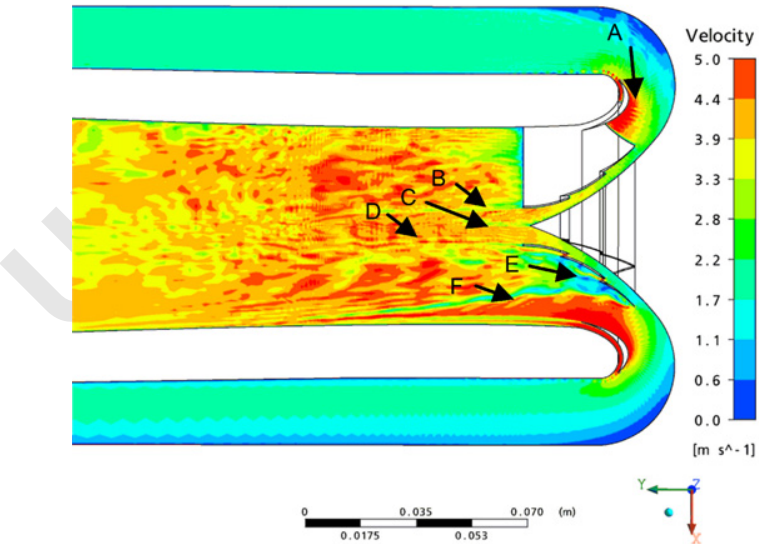

flow through the narrow gaps (annotated region E in Fig. 2a), can be coupled with vibrations modes of an adjacent structure, i.e. flow vanes BEW can be referred to as a turbulent liquid jet (annotated region $C$ in Fig. 2a), a periodic modulation of a coaxial liquid layer can generate periodically varying structures in the jet and in the shear layer (annotated regions B and D in Fig. 2a), which can also resonate with natural vibrations of the flow vanes and thus cause a positive feedback,

- The vortex streets (see region G on Fig. 2b), whose existence can be annotated by using shear strain rate isosurfaces [1], are generated in the wake of a flow vane close to the guide tube and in the wake of vane supports. They give rise to another feedback and could cause fluid-induced vibrations of the structure.

This possibility of fluid-structural coupling was investigated theoretically by Samec et al. [6]; initial indications showed that $\mathbf{Q 6}$ the flow vanes could fail but not the hull or structure as a whole. The matter was of sufficient concern to warrant a very thorough experimental program with sensors dedicated to monitor areas deemed to be particularly critical so as to back up the theoretical calculations with actual proof.

The flow vanes assembly underwent a very rapid development. Thus, rather than assembling the flow vanes using a dedicated fixture, an ad-hoc welding procedure was adopted. This led to a number of weaknesses in the welding pointed out in Fig. 3, which explains the ultimate failure of the vanes. A coupled dynamic analysis performed ahead of the test $[5,6]$ showed that even if the original fully welded design had been retained, fatigue failure would have still remained a serious concern for a prolonged usage. Thus, any future work involving the flow vanes should start with a renewed effort at improving strength and manufacturing technology of the vanes. Nevertheless, the tested design collected sufficient data to allow the influence of the flow vanes to be assessed, with no detrimental effect on safety.

The vibration loads on the flow vanes that lead to failure of the welds were clearly registered during the experiment by the acceleration sensors and microphone; however, the structure of the target as a whole remained steady and unaffected by vibration up to the point where one of the vanes broke off and impacted the hull. The remainder of the flow vanes, located along the beam window, remained intact as their assembly had benefited from a more comprehensive welding.
- Since the flow regime that occurs in the central zone of the

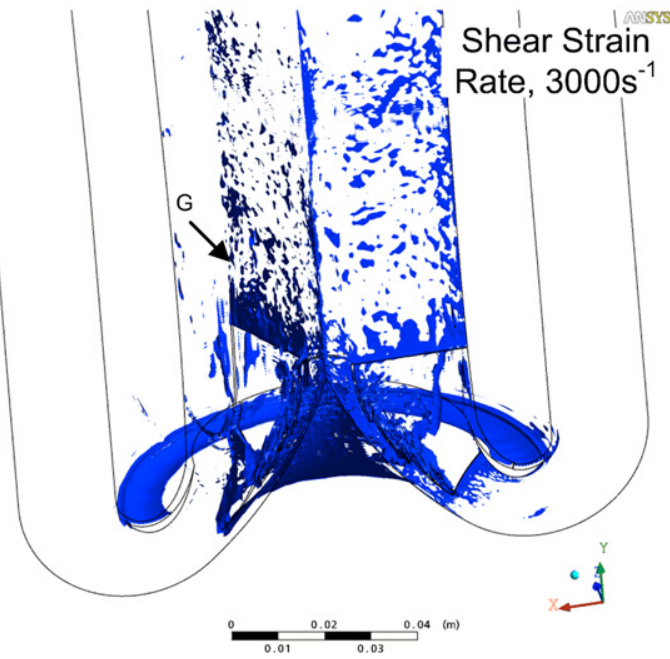

Fig. 2. LES calculations: (a) instantaneous velocity field and (b) shear strain rate annotate regions in flow field where flow-induced instabilities can occur. 

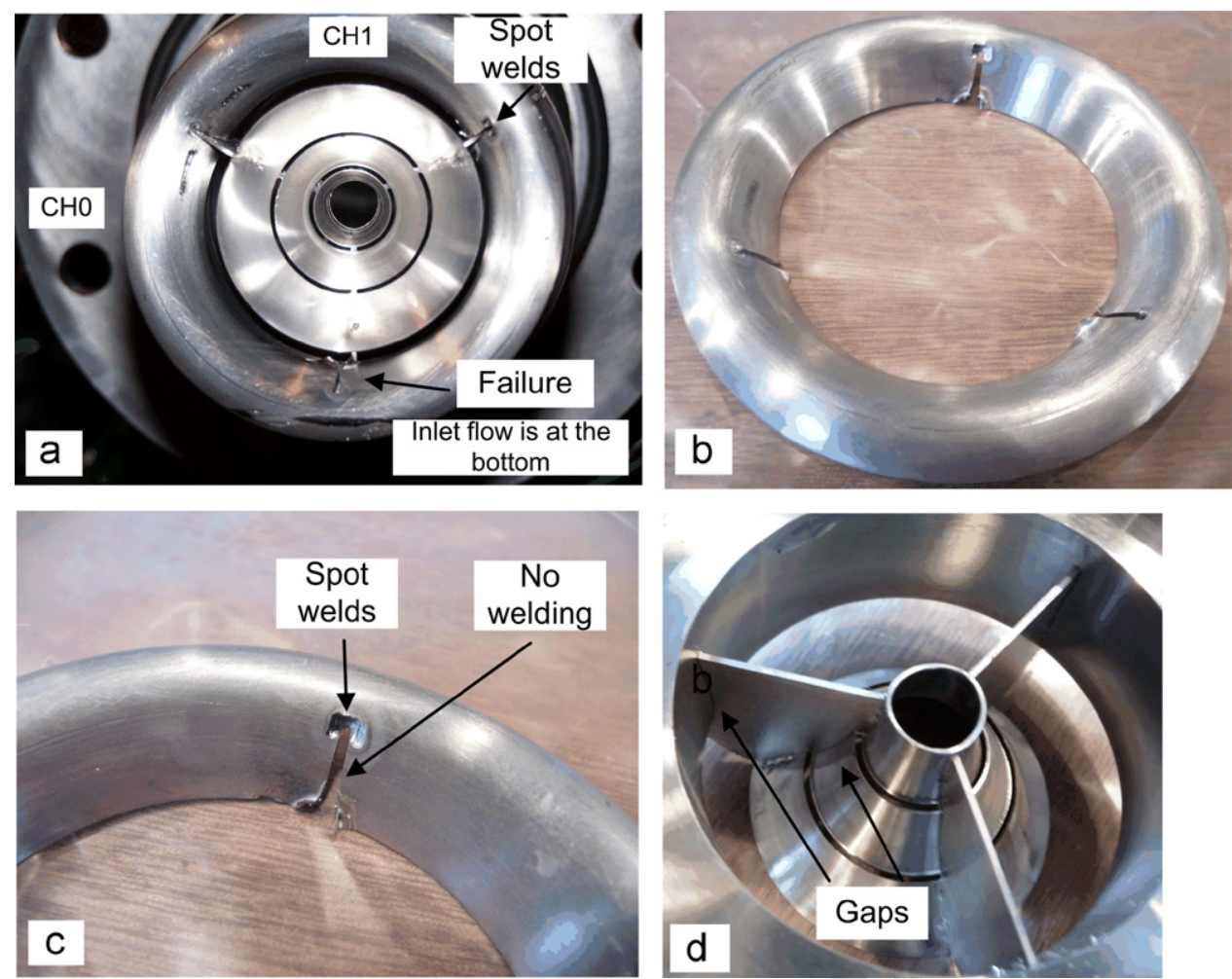

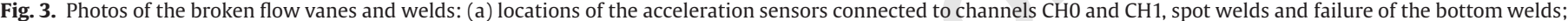
(b) and (c) detached flow vane; and (d) the gaps between blades and supporting ribs.

Q7 During the first day of operation, a part of clear tones and cavitation noise, which were also registered by the personnel in the laboratory for regimes with developed cavitation, structural accelerations with moderate amplitudes, were acquired. The mock-up was operated for about $1 \mathrm{~h}$. During the second day, just after short operation (about $25 \mathrm{~min}$ ) at moderate and higher flow rates, a very loud crackling and fluttering noise announced the failure of the vanes and the end of the experiment. Photos of the flow vanes and welds after the failure are shown in Fig. 3.

In this paper, we show unique data of the resonant structural response characteristics, which point out the weaknesses of the manufacturing and design of flow vanes. Advanced time-frequency analysis has been used to detect flow regimes wherein flow-excited acoustic or structural resonances appear. During the first stage of the experiment when the configuration without flow vanes was tested, neither peaks nor acoustic and/or structural resonances in the acceleration data sets have been detected [7].

\section{The experiment}

The results presented here refer to the METEX 1 experiment. The main goal of METEX 1 was to investigate the hydraulic and structural behavior of the EURISOL target mock-up under various flow conditions and for two flow configurations, with and without flow vanes. A detailed description of the experimental set-up together with results on the observed global structural behavior is given in Refs. [5,7].

During the experiments reported here for the configuration with flow vanes, the pressure of the cover gas, the total mass flow rate, the pressure loss in the mock-up, temperatures at various locations, structural acceleration, strain and sound data were acquired at the most interesting locations (see Ref. [7]). Schematic of the experimental set-up is given in Fig. 4.

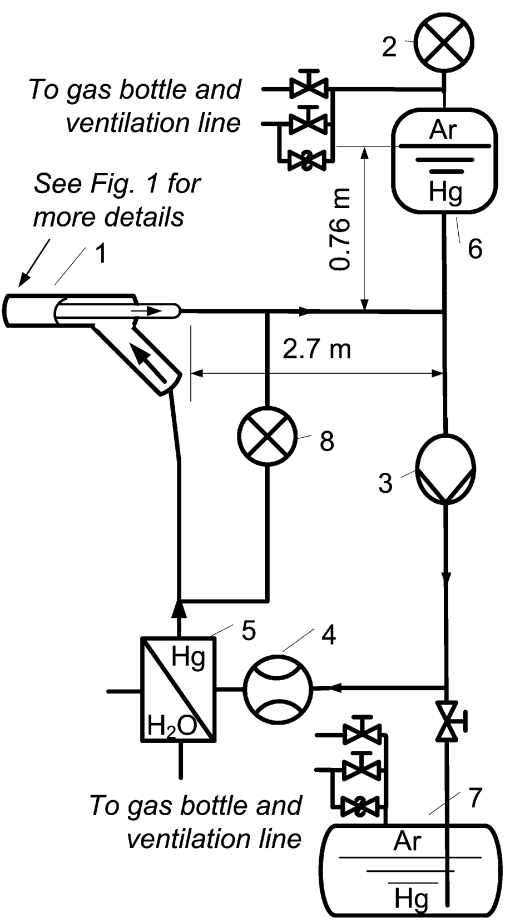

Fig. 4. Experimental set-up: 1-mock-up, 2-manometer-cover gas pressure, 3-electromagnetic pump, 4-flow meter, 5-heat exchanger, 6-expansion tank, 7-storage tank and 8-differential manometer-pressure loss measurement.

All tests were conducted at the IPUL mercury loop (Fig. 5), which has DN100 piping assembled in a vertical plane on a frame. The target mock-up is connected to the loop interface flanges. During normal operations in a dedicated facility the target would be rigidly supported by the interface to the loop; on the laboratory 

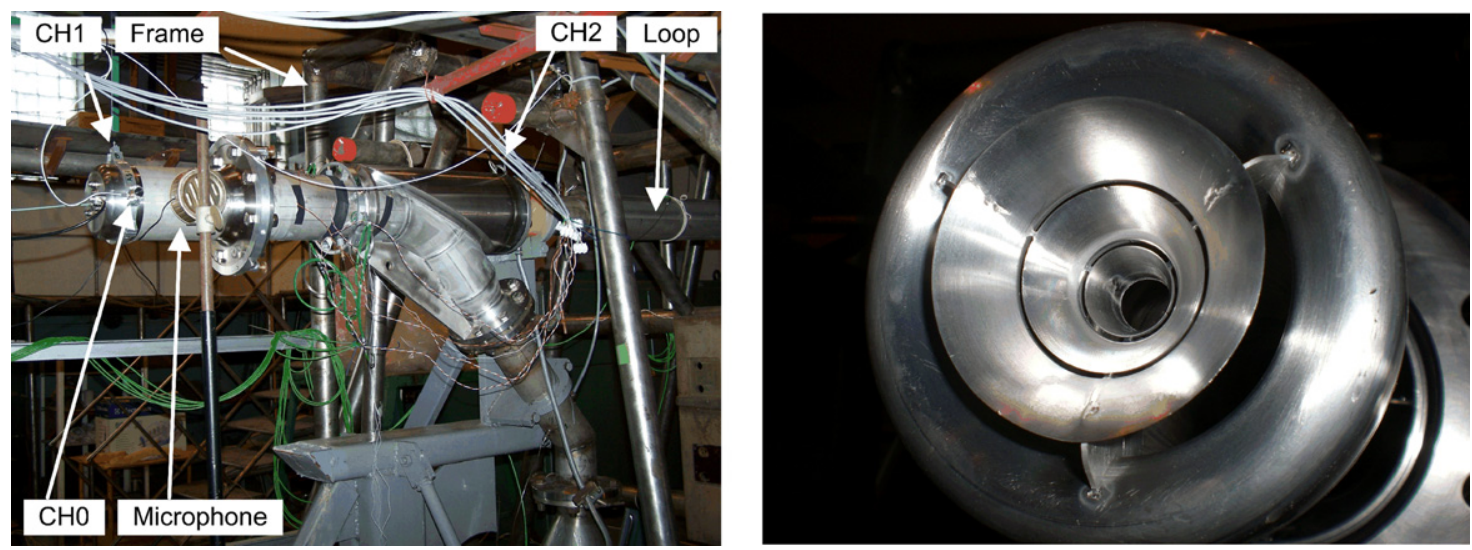

Fig. 5. Photo of the set-up shows sensors, microphone, IPUL loop, loop frame (left) and flow vanes (right).

test loop this was reproduced by a supporting truss bolted to the laboratory floor that represents less-favorable conditions and is, therefore, conservative. The maximum liquid metal flow rate achieved with the configuration without flow vanes was about $10 \mathrm{l} / \mathrm{s}$.

As during the pump operation, up to $40 \mathrm{~kW}$ of thermal power is dissipated into the liquid metal, four cooling water jackets on the stainless steel piping served to remove deposited heat. The cooling water flow rate through all four heat exchangers was manually regulated. Therefore, the mercury temperature in the loop was kept in a range from 3 to $36{ }^{\circ} \mathrm{C}$. The total mass of the mock-up filled with mercury was $176 \mathrm{~kg}$.

The instrumentation used for hydraulic and structural test included the following:

- three acceleration sensors (KISTLER 8632C5), which are fixed to the target mock-up as shown in Fig. 5. Structural acceleration of the target window was measured by $\mathrm{CHO}$ (horizontal component) and $\mathrm{CH} 1$ (vertical component) and of the target mock-up at the outlet interface flange was measured by $\mathrm{CH} 2$ (vertical component of the acceleration);

- the conduction-type electromagnetic flow meter (calibrated with a Venturi tube) was used for measuring the liquid metal flow rate. The relative error was 3\% of the maximum flow rate;

- the differential manometer (YOKOGAWA EJA110, position 8 in Fig. 4), which was used to measure the hydraulic pressure loss in the target mock-up. The relative error was about $2 \%$ of the full range (2.5 bar);

- the manometer (WIKA UT10, position 2 in Fig. 4), which was used to measure the pressure of the cover gas in the system. The static pressure in the system was adjusted in the range from 1 to 6.5 bar. The minimum static pressure in the system was 1 bar due to the height difference of approximately $0.76 \mathrm{~m}$ between the mercury level in the expansion tank and the target;

- the strain gauges attached to the hull at points designed to monitor bending of the hull in the vertical and horizontal planes as well as two points on either side of the critical weld connection from the hull to the support (see Section 4.2.1.2).

The acceleration, pressure, flow rate and pressure loss data were acquired by using both low-speed (National Instruments FIELDPOINT blocks, at $1 \mathrm{Sample} / \mathrm{s}$ ) and high-speed (Digital LeCroy Oscilloscope, at 5000 Samples/s) data acquisition systems. They were collected into a single data base. Sound data are acquired by a personal computer at $22.5 \mathrm{kHz}$. The strain data were acquired at $1200 \mathrm{~Hz}$ by SPIDER 8 system (HMB-Hottinger Baldwin Messtechnik) into the different data base.
Various phenomena and instabilities, which are partly mentioned here and extensively investigated in computational studies [5,6], generate forces, which directly cause accelerations and displacements of the target mock-up and can be registered by means of various time-frequency analyses of structural acceleration, sound or strain measurements.

As during the METEX experimental campaign it is important to extract hidden transient system behavior and as the Fourier transform only provides the information in the frequency domain, the advanced time-frequency analysis is employed. Mainly, the short-time Fourier transform (STFT) [8] is used.

\section{Test conditions}

The first test performed is a vibration test for no-flow condition. It consisted of two essential parts: a noise and a dampened contribution. The dampened contribution resulted from the application of an external force (generated by a hammer) to initiate vibrations. The tests are preformed at the start and end of 2-day sessions. The spectrograms (Hanning window 64 samples, 512 frequency bins and 14 time bins) of the acceleration signal during the vibrations tests (Fig. 6) reveal clearly in the time-frequency domain that the system response was changed after the first day of operation and at the end of the experiment.

The variation of the liquid metal flow rate, the pressure loss, the pressure of the gas in the expansion tank and temperatures at the inlet and at the outlet of the mock-up are presented in Figs. 7 and 8 during 28.01.2009 and 29.01.2009, respectively. In order to analyse efficiently the huge amount of acceleration and sound data, which were acquired at a sampling rate of $5000 \mathrm{~Hz}$ during $2 \mathrm{~s}$ and at $22.5 \mathrm{kHz}$ during $60 \mathrm{~s}$, respectively, the complete experimental data base created during these 2 days was divided into several blocks, as presented in Tables 1 and 2 in Section 4. Absolute times and brief comments accompany information given in Figs. 7 and 8. In any case, since the final failure event (No. 3) is taken as a reference, an attempt is made to follow the cause of failures and to highlight crucial events.

\subsection{Welds failure}

The failure event, which is annotated as Event 3 in Fig. 8, remains the reference. At about 12:45:17 the total pressure loss increased by about 500 mbar, whereas the flow rate dropped from 9.94 to $8.82 \mathrm{l} / \mathrm{s}$. The sound level registered by the microphone increased almost instantaneously as shown in Fig. 9a. The acquisition of the sound pressure started at 12:45:00 at the 
a

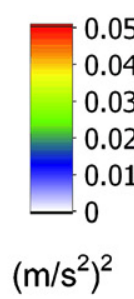

b

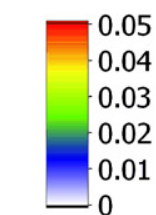

$\left(\mathrm{m} / \mathrm{s}^{2}\right)^{2}$

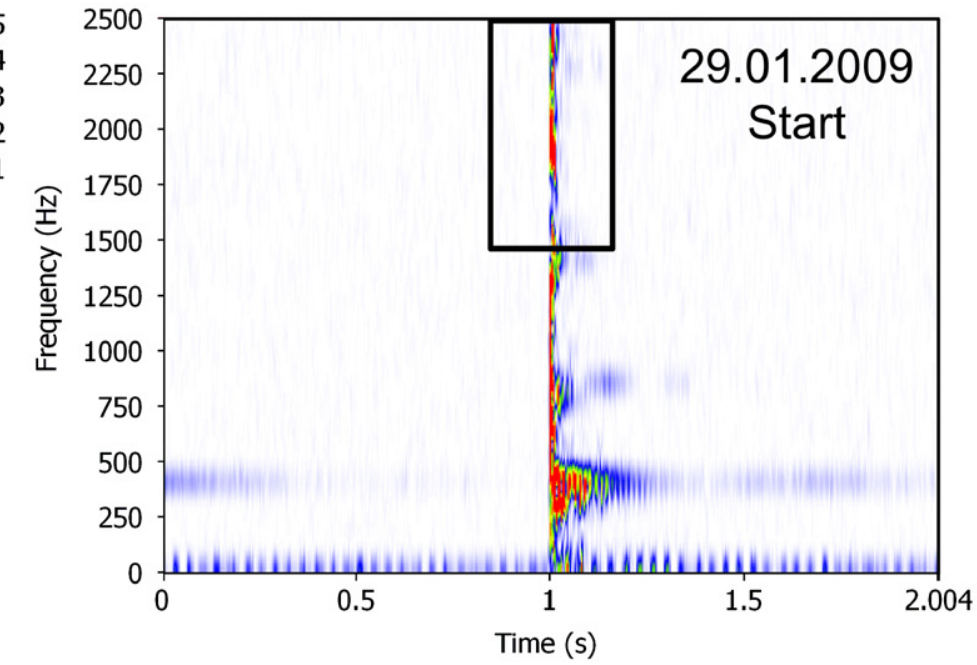

C
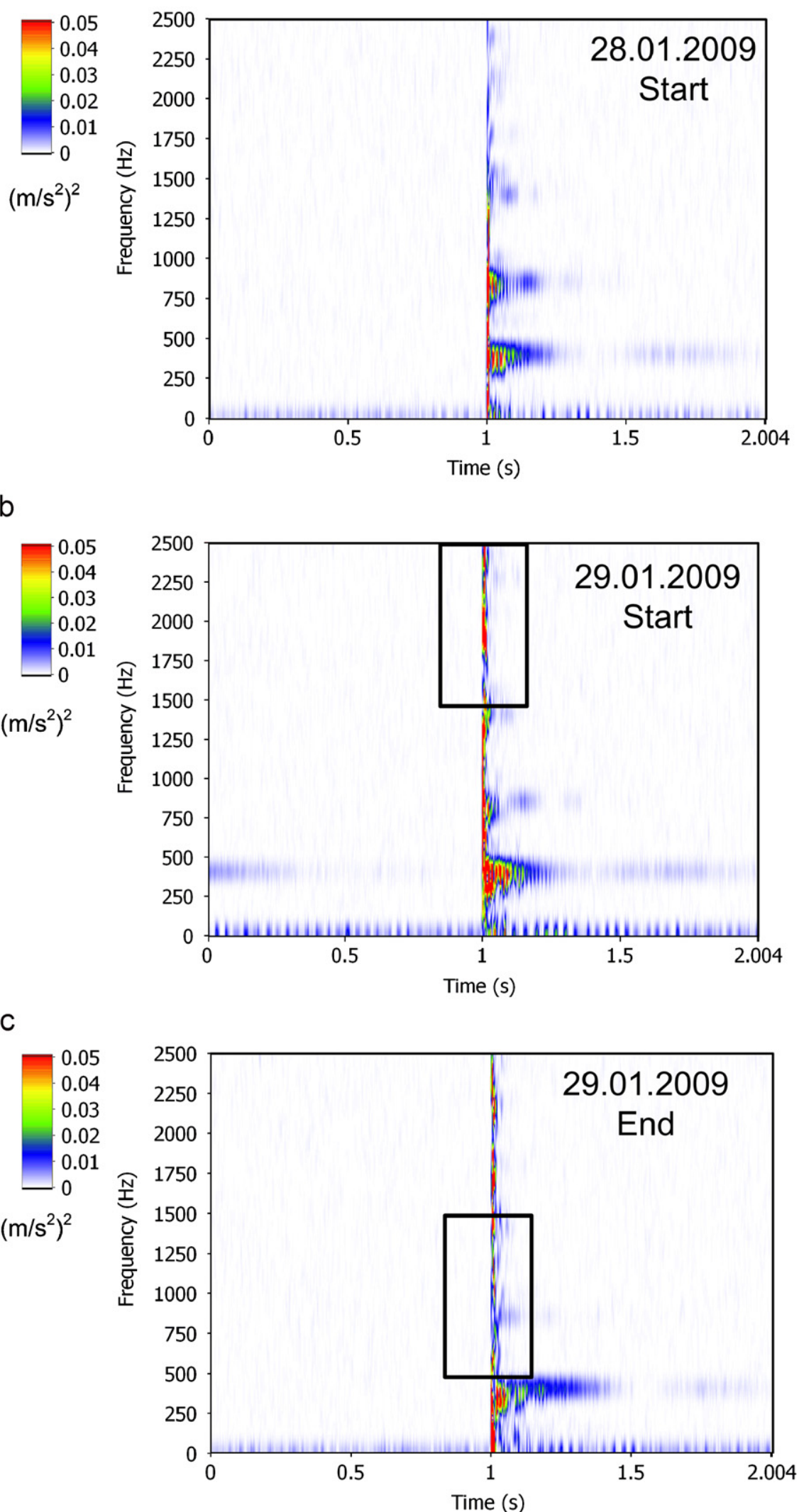

67

69

71

73

75

77

79

81

83

85

87

89

91

93

95

97

99

101

103

105

107

109

111

112

113

114

115

116

117

118

119

120

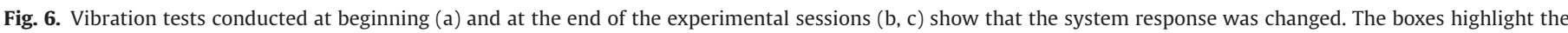
existence of high-frequency components, which did not exist at the beginning of the experiment but appeared when the first day session was finalized. 
a

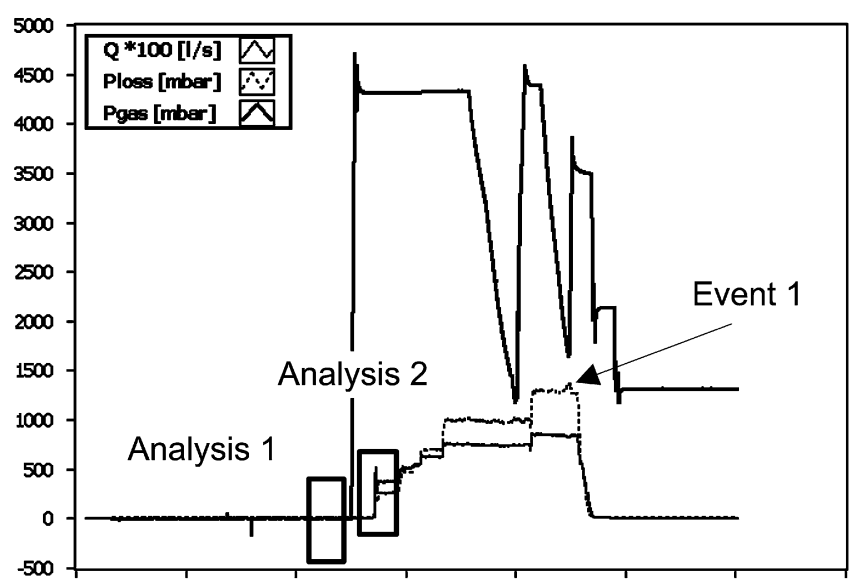

13:45:00.000 14:00:00.000 14:15:00.000 14:30:00.000 14:45:00.000 15:00:00.000 15:15:00.000 15:30:00.000 $\begin{array}{llllllll}28.01 .2009 & 28.01 .2009 & 28.01 .2009 & 28.01 .2009 & 28.01 .2009 & 28.01 .2009 & 28.01 .2009 & 28.01 .2009\end{array}$ Time b

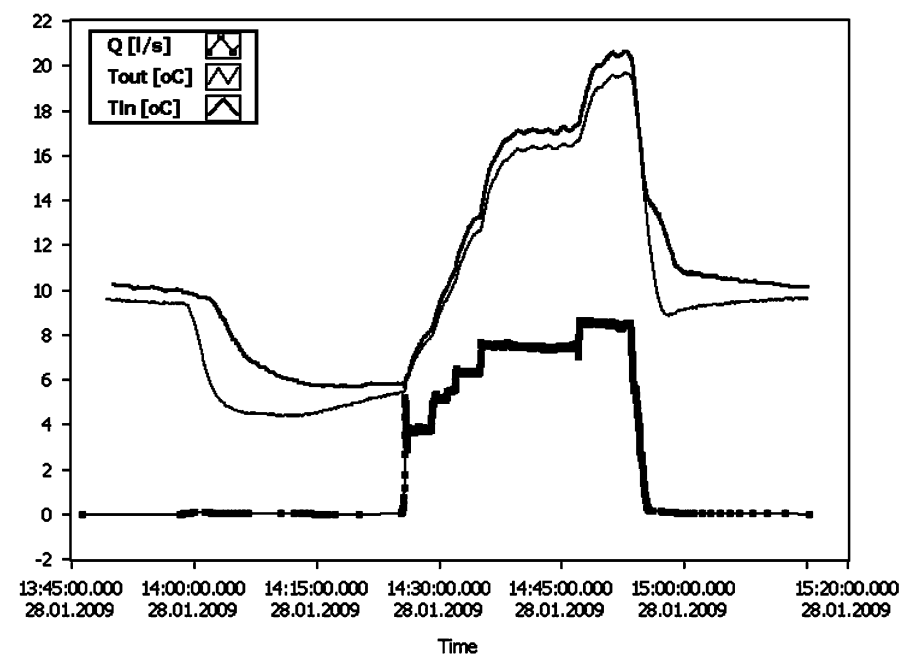

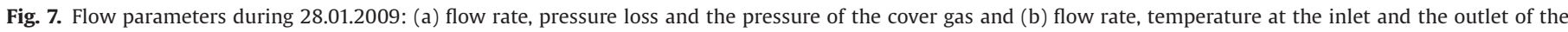
mock-up.

b

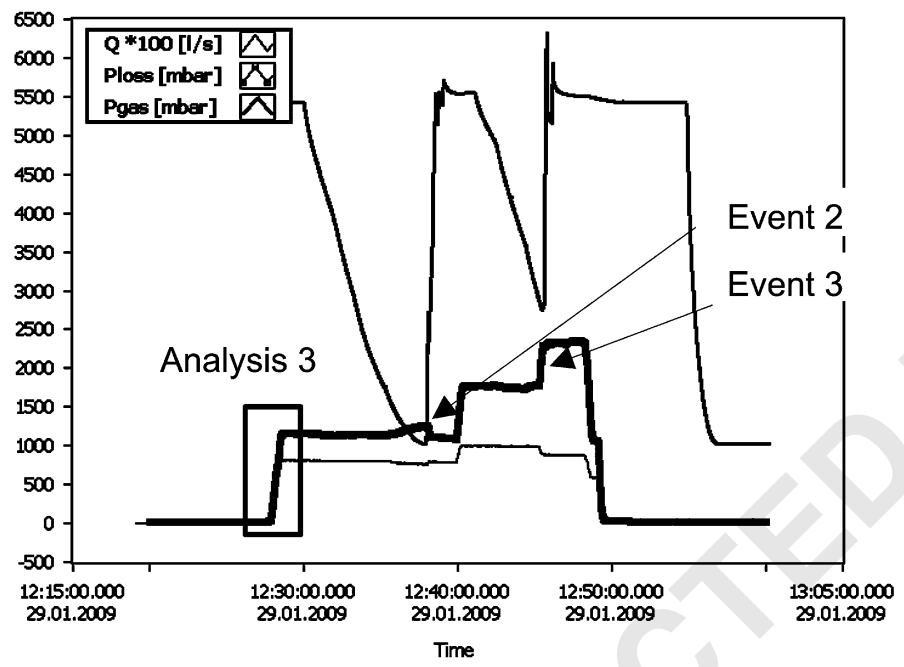

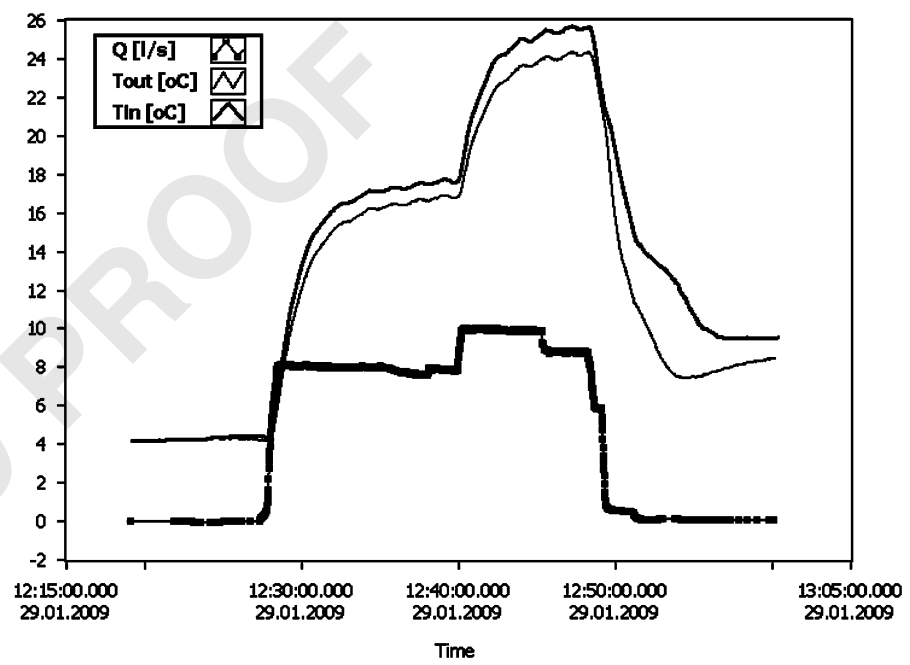

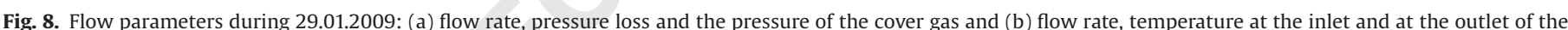
mock-up.

Table 1

Test matrix for 28.01.2009.

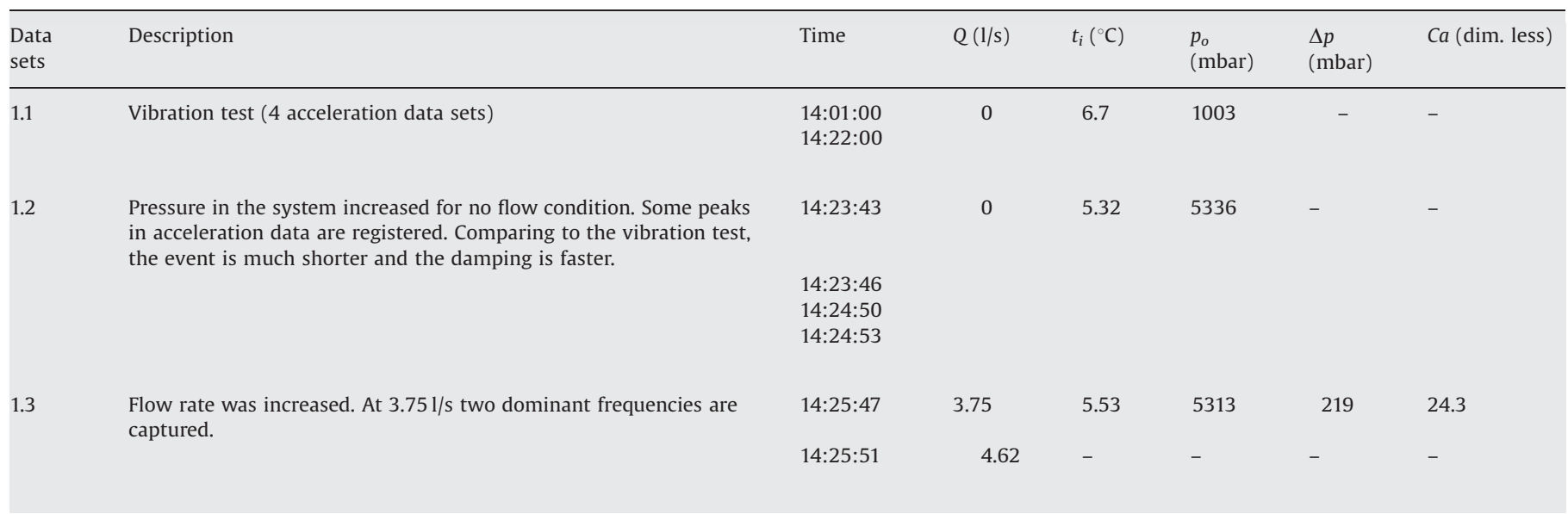


1

Table 1 (continued)

\begin{tabular}{|c|c|c|c|c|c|c|c|}
\hline $\begin{array}{l}\text { Data } \\
\text { sets }\end{array}$ & Description & Time & $Q(1 / s)$ & $t_{i}\left({ }^{\circ} \mathrm{C}\right)$ & $\begin{array}{l}p_{o} \\
\text { (mbar) }\end{array}$ & $\begin{array}{l}\Delta p \\
\text { (mbar) }\end{array}$ & $\mathrm{Ca}$ (dim. less) \\
\hline \multirow[t]{2}{*}{1.4} & $\begin{array}{l}\text { Flow rate was reduced below } 3.75 \mathrm{l} / \mathrm{s} \text {. Both frequency components } \\
\text { disappear. }\end{array}$ & $14: 26: 01$ & 3.75 & 7.1 & 5313 & 259 & 20.5 \\
\hline & & $14: 26: 03$ & - & - & - & - & - \\
\hline \multirow[t]{2}{*}{1.5} & $\begin{array}{l}\text { Flow rate was increased above } 3.75 \mathrm{l} / \mathrm{s} \text {. Both frequency components } \\
\text { can be again seen. }\end{array}$ & $14: 26: 16$ & 3.75 & 7.1 & 5313 & 259 & 20.5 \\
\hline & & $14: 28: 56$ & 3.73 & 7.86 & 5312 & 261 & 20.4 \\
\hline \multirow[t]{2}{*}{1.6} & Constant flow rate and the pressure in the system & $14: 31: 00$ & 5.49 & 10.1 & 5318 & 529 & 10.4 \\
\hline & & $14: 32: 00$ & 5.52 & 9.84 & 5319 & 529 & 9.8 \\
\hline \multirow[t]{2}{*}{1.7} & Constant flow rate and the pressure in the system & $14: 32: 16$ & 6.33 & 11.9 & 5323 & 703 & 7.6 \\
\hline & & $14: 35: 00$ & 6.26 & 11.3 & 5322 & 697 & 7.6 \\
\hline \multirow[t]{2}{*}{1.8} & Constant flow rate and the pressure in the system & $14: 35: 17$ & 7.54 & 14.5 & 5331 & 997 & 5.34 \\
\hline & & $14: 38: 13$ & 7.58 & 14.5 & 5330 & 997 & 5.34 \\
\hline \multirow[t]{2}{*}{1.9} & Constant flow rate and the pressure in the system & $14: 47: 21$ & 8.54 & 17.4 & 5389 & 1290 & 4.17 \\
\hline & & 14:48:07 & 8.55 & 17.6 & 5389 & 1297 & 4.15 \\
\hline \multirow[t]{2}{*}{1.10} & For a constant flow rate, the pressure of the cover gas was reduced. & $14: 52: 17$ & 8.29 & 19.6 & 2659 & 1361 & 1.95 \\
\hline & & $14: 52: 21$ & - & - & - & - & - \\
\hline
\end{tabular}

Dim. less $=$ dimension less.

Table $\mathbf{2}$
Test matrix for 29.01.2009.

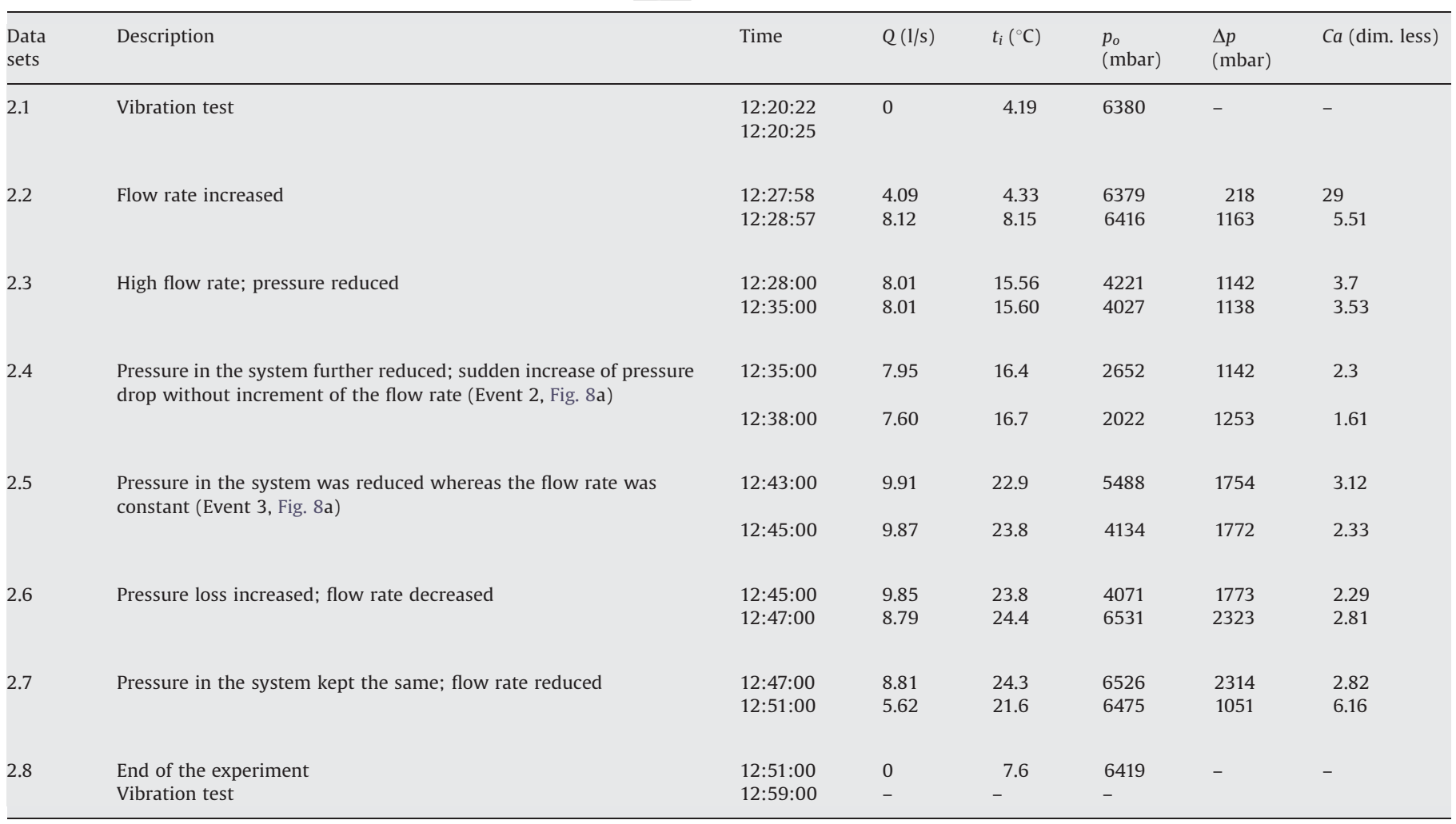

Dim. less $=$ dimension less. 


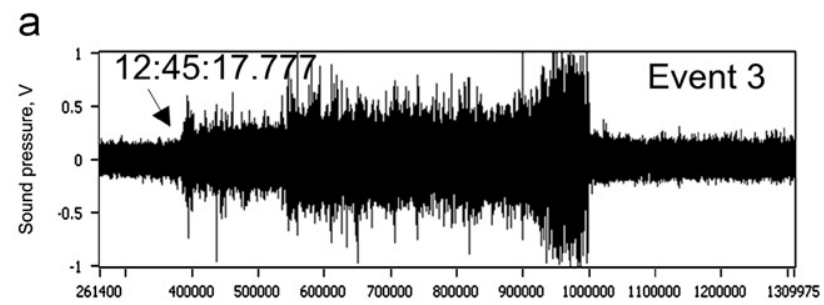

9

11

b

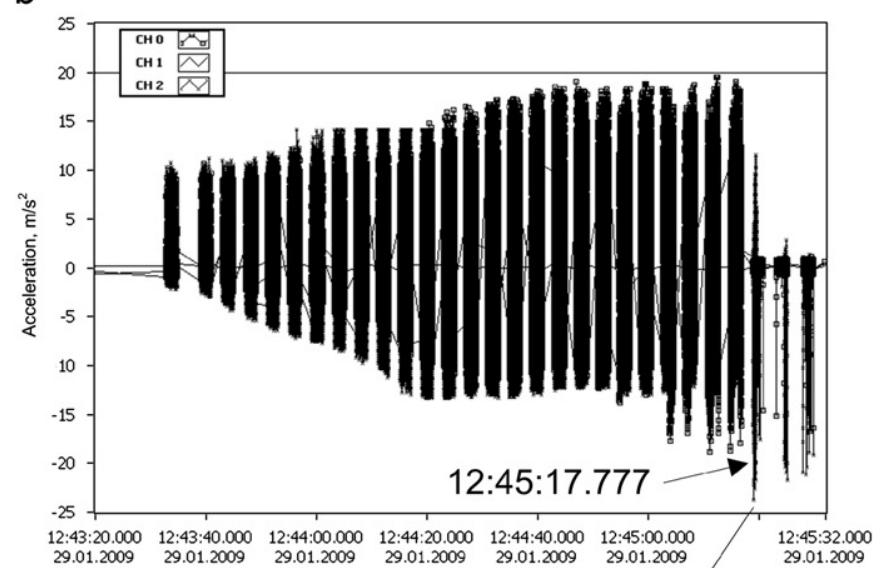

C

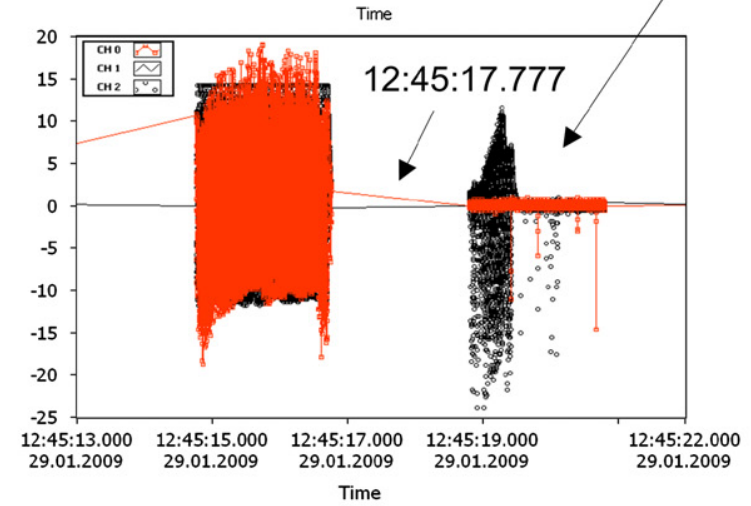

d

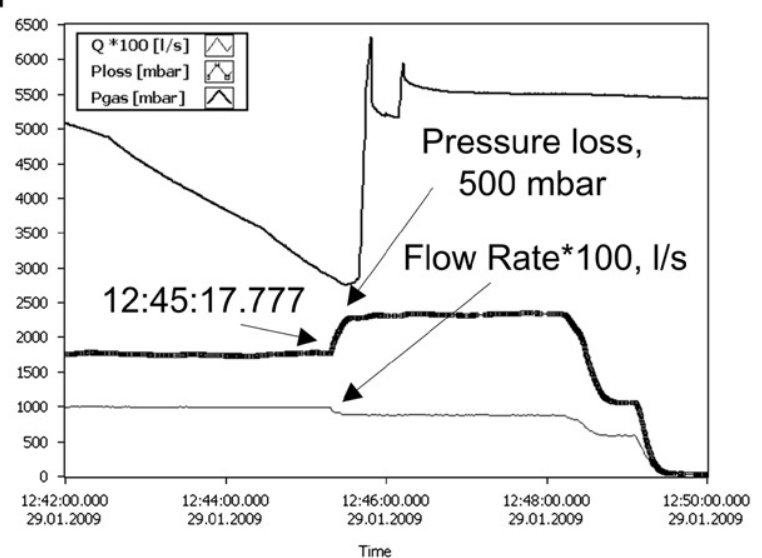

Fig. 9. Signals acquired during time interval when final failure (Event 3 ) occurred: (a) sound pressure; (b) and (c) acceleration; and (d) pressure loss, flow rate and pressure of the cover gas. Arrows indicate absolute time when failure occurred.

sampling rate of $22.5 \mathrm{kHz}$. Five minutes after the final failure was detected, the experiment was safely terminated.

The following signals (Fig. 9) are a clear indication of an unexpected event; they show the amplitude enhancement at the time when welds are assumed to have been broken and the blade separated from the supports:

- Sound pressure (Fig. 9a) results at about 12:45:00 show sudden and huge increase of the sound level in the laboratory. The amplitude is given in $\mathrm{V}$ (Volts). The sampling rate was $22.5 \mathrm{kHz}$. The acquisition started at 12:45:00.

- Acceleration results (Fig. 9b) between 12:43:00 and 12:45:00 show an increase of a factor of two during $2 \mathrm{~s}$. During this period the flow rate was kept the same, but the pressure of the cover gas was reduced. The pressure loss remained constant. Previous results presented by Milenkovic [7] showed that the regime of developed cavitation was reached for the given flow conditions at 12:43:00. Therefore, the amplitude increase is caused by developed cavitation and the contribution due to the structural resonance is hidden in the noise,

- Acceleration results (Fig. 9c) at 12:45:17.177 show that acceleration amplitudes drop after welds were broken and the blade was freed.

- Pressure loss was increased for about 500 mbar, whereas flow rate decreased slightly (Fig. 9c).

In order to systematise and search systematically for crucial events that led to failure of the welds, a time-frequency analysis was conducted for all data sets presented in Tables 1 and 2. The acceleration data contained in a single set are acquired for $2 \mathrm{~s}$ at $5000 \mathrm{~Hz}$. The flow characteristics, which are given in Tables 1 and 2 at discrete times, are averaged during time intervals of $2 \mathrm{~s}$. The most important comments are given in the column with a headline entitled "Description". For easier tracking, important events and segments used for analysis are denoted "Event" or "Analysis" in Figs. 7a and 8a and included in corresponding figures. The Cavitation $\mathrm{Ca}$ number is defined as $\mathrm{Ca}=\left(p_{o}-p_{\text {sta }}(t)\right)$ / $\Delta p$, where $p_{\text {sta }}$ is the pressure loss and $p_{o}$ is the pressure in the $\mathbf{Q 8}$ system at the outlet of the mock-up. The pressure loss is determined experimentally. Basics of the implemented methods and most important results are given in the next section.

\section{Experimental results and discussions}

The results presented in the next subsections follow cause of events.

\subsection{Pressure increase without pump operation}

At the beginning of the experiment, the pressure of the cover gas in the system was increased to 5336 mbar without pump operation (Fig. 10a). The peaks (Fig. 10b and c) have been registered in the acceleration data set 1.2 (see Table 1) but without any external excitation. Comparison with vibration tests reveals much shorter time duration and higher damping of the signal. Therefore, any unknown external source of such behavior is excluded. Similar peaks were also registered in several flow regimes (without external excitation and with pump operation) before welds failure occurred (see Fig. 11a and b). The spectrograms (Hanning window 64 samples, 512 frequency bins and 14 time bins) of the acceleration signal show the characteristics of detected peaks in the time-frequency domain (Fig. 10c and d). Total number of samples was 215, which corresponds to $2.004 \mathrm{~s}$. The sensor at vertical position $(\mathrm{CH} 1)$ detected the peaks, whose spectral power is spread over full frequency range (in particular, strong components at about 1000 and $1750 \mathrm{~Hz}$ ), whereas the sensor at horizontal position $(\mathrm{CHO})$ has detected only a weak peak whose spectral power is well-localized 
a

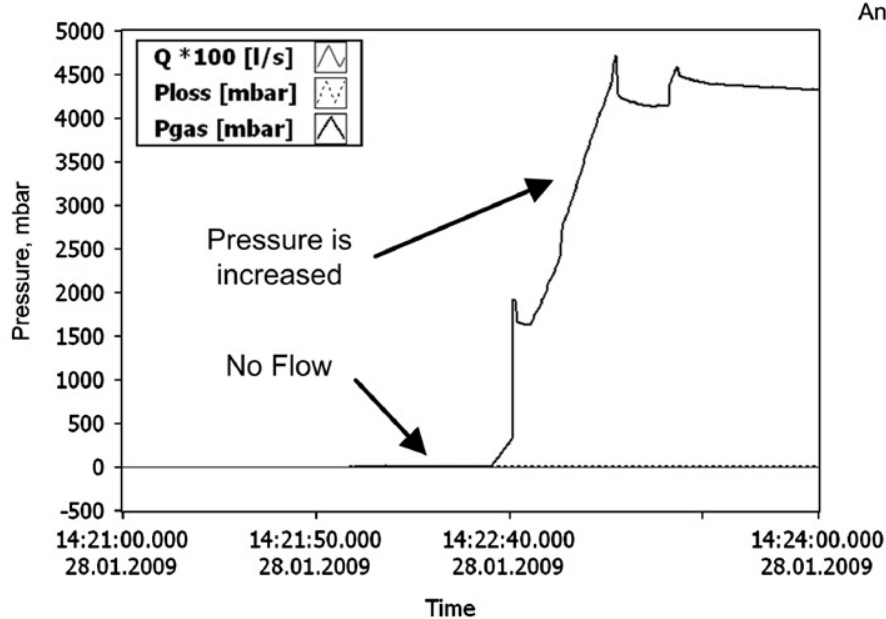

C

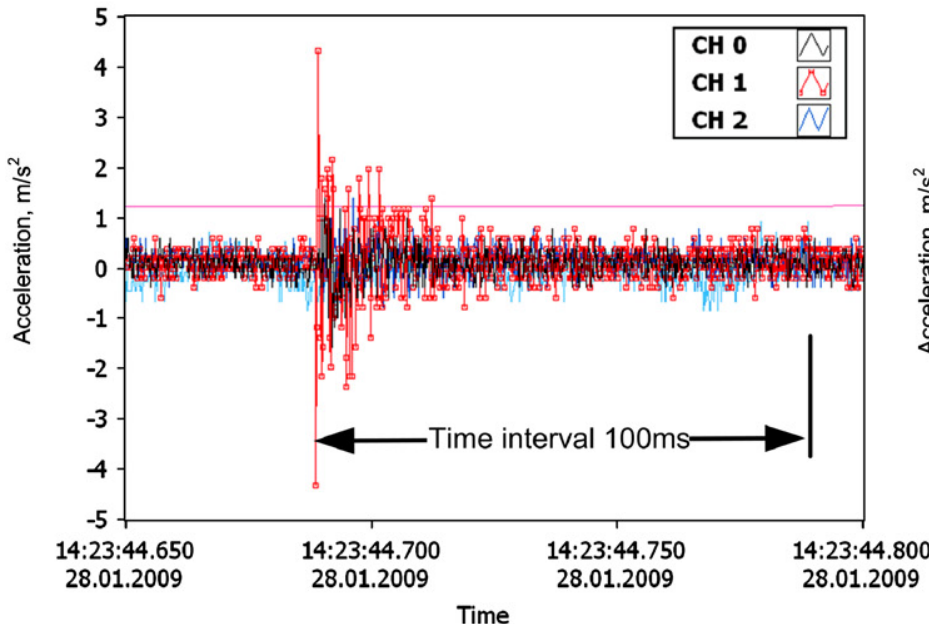

d b
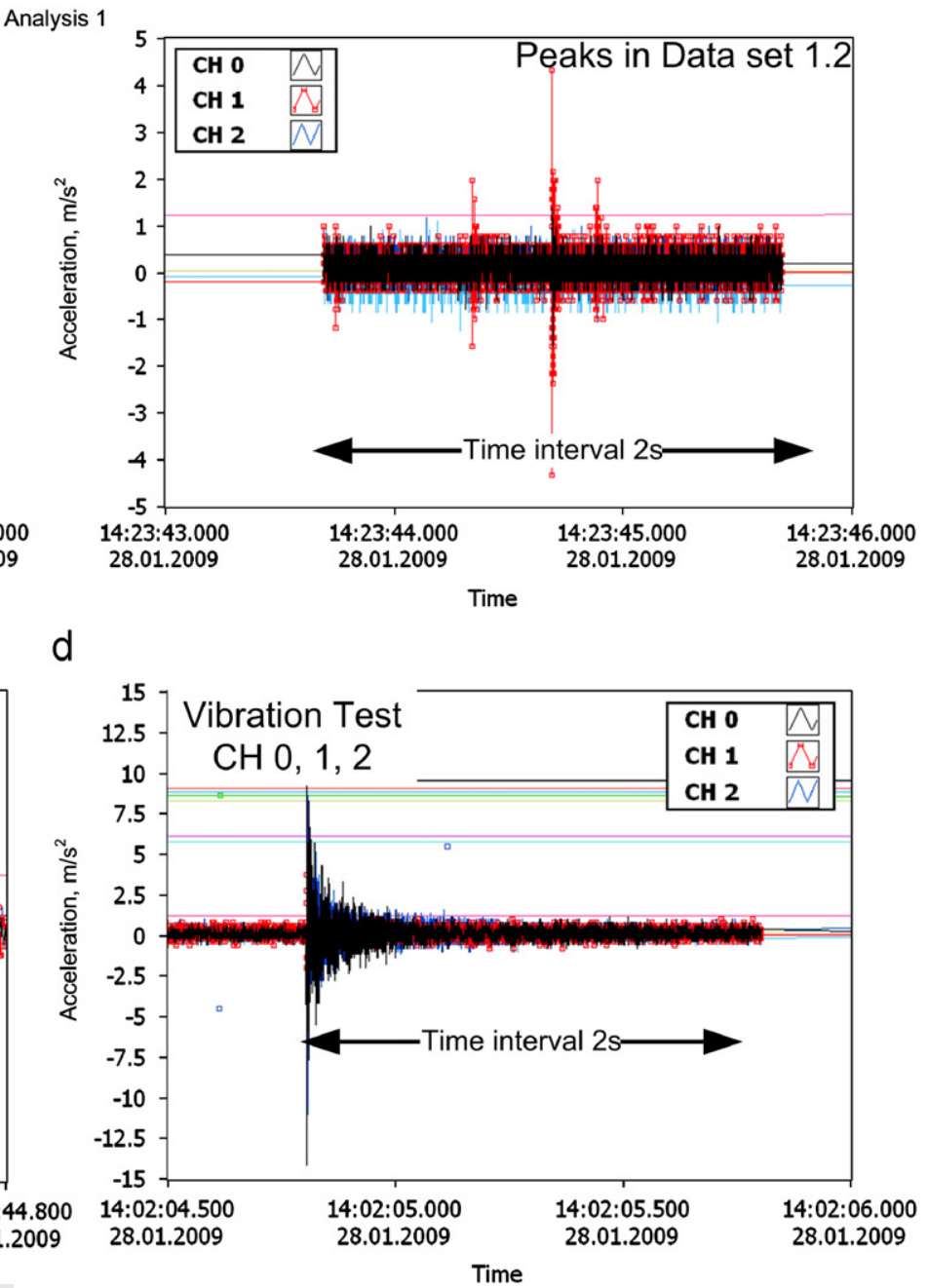

67

69

71

73

75

77

79

81

83

85

87

89

91

93

95

97

99

101

Fig. 10. Flow condition (a), acceleration data (b), zoomed peak in acceleration data (c) and vibration test (d) at beginning of the experiment. The pressure of the gas was increased to about 4500 mbar. Several peaks are registered by all sensors.

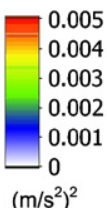

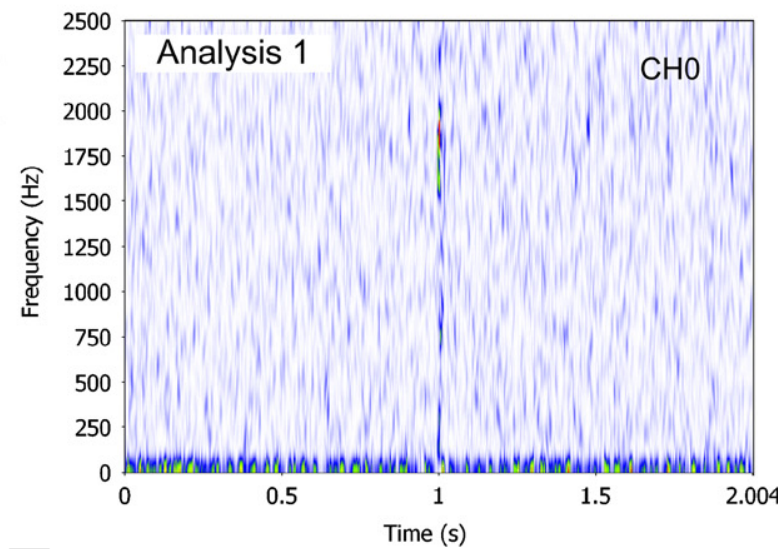

b

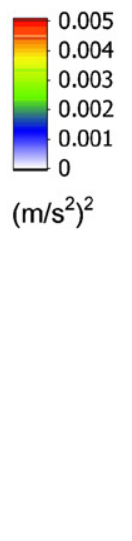

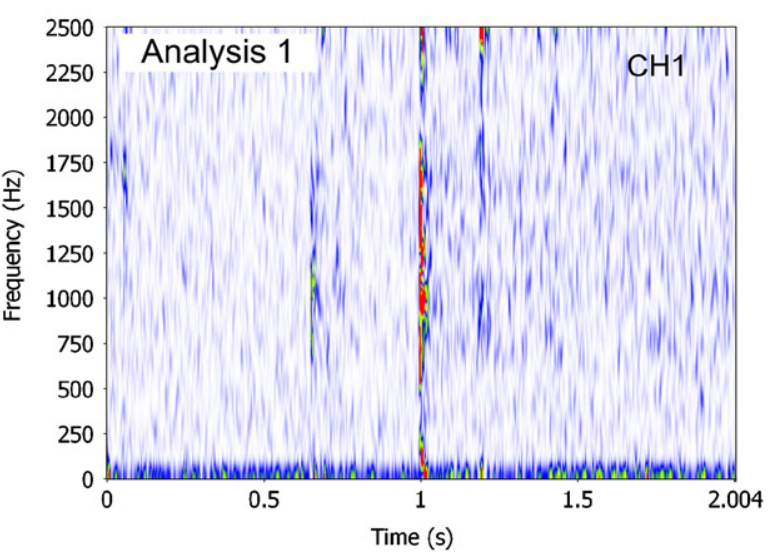

Fig. 11. Spectrograms of detected peaks show how sensors at different locations react: (a) the acceleration sensor at vertical position (CH) detected two consecutive events, whereas the sensor at horizontal position ( $\mathrm{CHO}$ ) detected a well-localized peak in a frequency domain at about $2 \mathrm{kHz}$.

in a frequency domain (at about $2000 \mathrm{~Hz}$ ). If the peaks corresponded to the cracks and if additional loads generated by the flow-induced vibrations or cavitation enhanced their growth and increase, the system behavior would have been changed and one would have expected a fundamental change in dynamics of the system. This fact can be confirmed based on vibration test results presented in Fig. 6. 
a

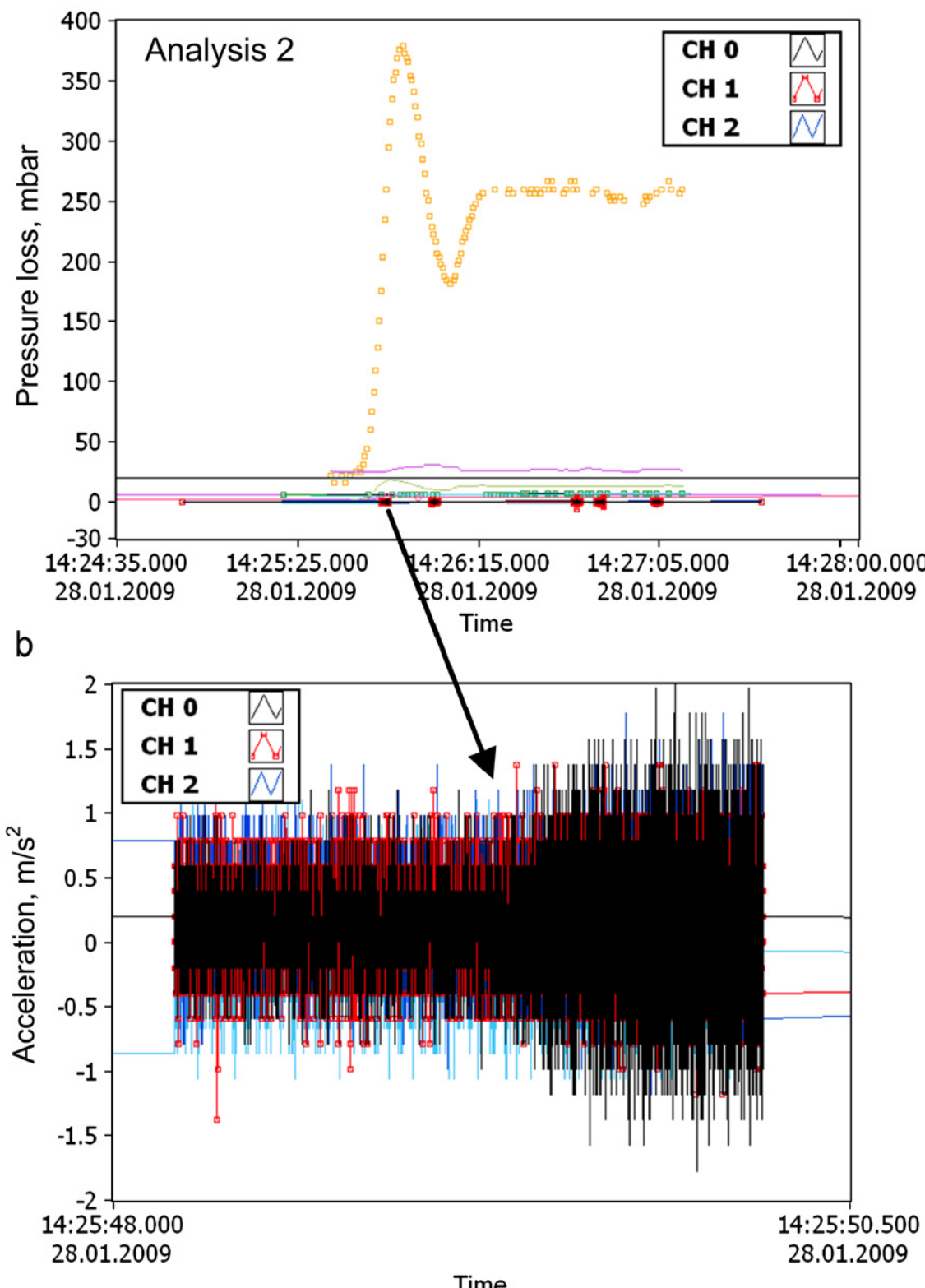

Fig. 12. As the flow rate was increased (a), a change in structural behavior can be seen in the acceleration data (b) - a slight increment in the amplitudes as annotated by the arrow. The pressure of the gas was constant (a), whereas the flow rate was increased from 0 to $4.62 \mathrm{l} / \mathrm{s}$, then reduced and again increased.

\subsection{Structural response during pump operation}

\subsubsection{The first test (28.01.2009)}

4.2.1.1. Monitoring the test with acceleration sensors. Though the peaks in acceleration data were observed, the experiment was continued and the flow rate was continuously increased from 0 to 4.62 l/s (see Fig. 12a, data set 1.3 , Table 1 ). Suddenly, a small change_slightly increased amplitudes_was registered in acceleration data from both sensors (see Fig. 12b). The spectrograms (see Fig. 13a) revealed two resonance frequencies, the first one at $1000 \mathrm{~Hz}$ and the second one at $1750 \mathrm{~Hz}$. The clear tones were also registered by the microphone and heard by personnel in the laboratory (the human ear can detect sounds up to $20 \mathrm{kHz}$ ). Usually, whistling sounds can be generated by flows through narrow gaps. As some of the gaps existed due to manufacturing weaknesses and some are part of the current design, it is not possible to isolate the cause of such system behavior. However, the appearance of such behavior is a clear indication that the design should in any case be reviewed if the work is to be pursued. Furthermore, as the flow rate was in the cause of actions reduced (data set 1.4, Table 1, see Fig. 12a), both resonance components disappeared (see Fig. 13b), but another peak was detected at lower frequency. After that $1000 \mathrm{~Hz}$ component appeared again in the data (data set 1.5 , $\mathrm{CHO}$, see Fig. 13c). In the cause of events another strong peak was registered. The time-frequency analysis of the next data set (data set 1.6, Table $\hat{1}$, see Fig. 13d) showed various powerful resonance components without any change of flow rate. Such system behavior indicates that captured peaks can be caused by cracks in some welds. Just 30 min after the mock-up was run at higher flow rates and lower system static pressure, an increase of 100 mbar in pressure loss during drop of flow rate indicated a failure in the system (annotated as Event 1, Fig. 7a).

In addition, the spectrograms of the acceleration data set from sensors $\mathrm{CH} 1$ (vertical component) and $\mathrm{CH} 2$ (close to the inlet 
a

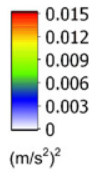

0.015
0.012
0.009
0.006
0.003
0
$\left(\mathrm{~m} / \mathrm{s}^{2}\right)^{2}$

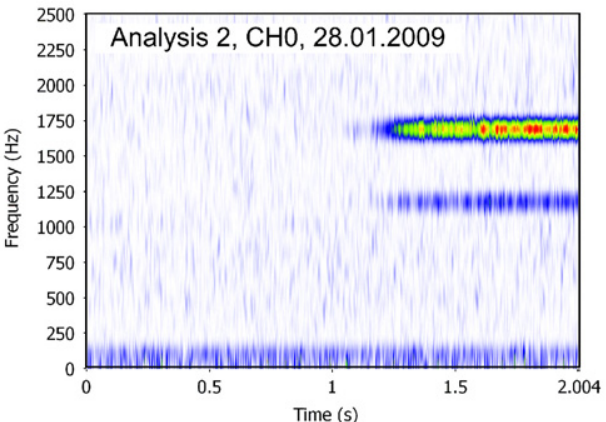

C
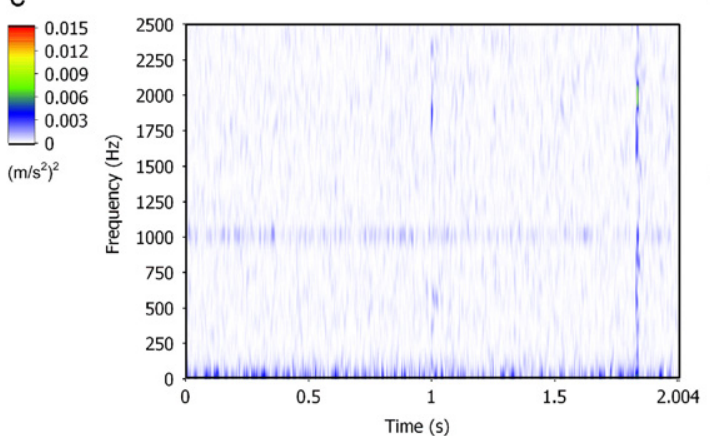

b

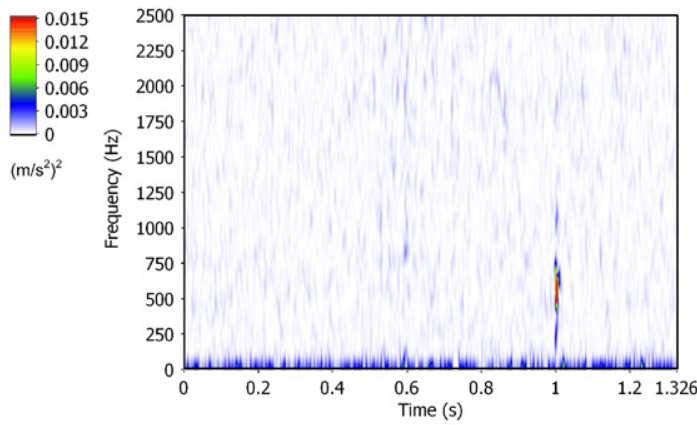

d

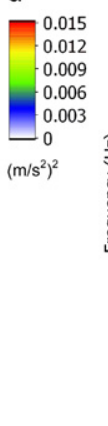

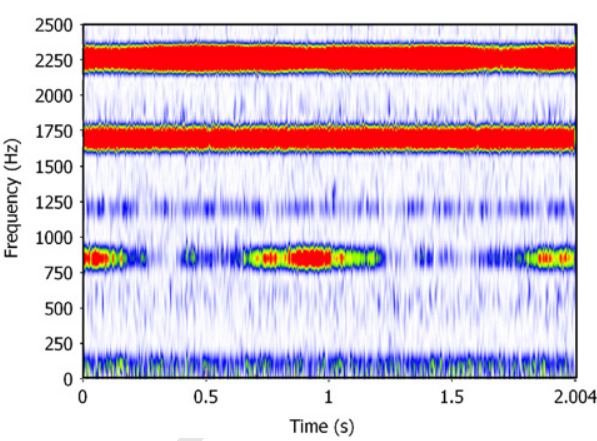

67

69

71

73

75

77

79

81

83

85

87

89

91

93

95

97

99

101

103

105

107

109

111

112

d
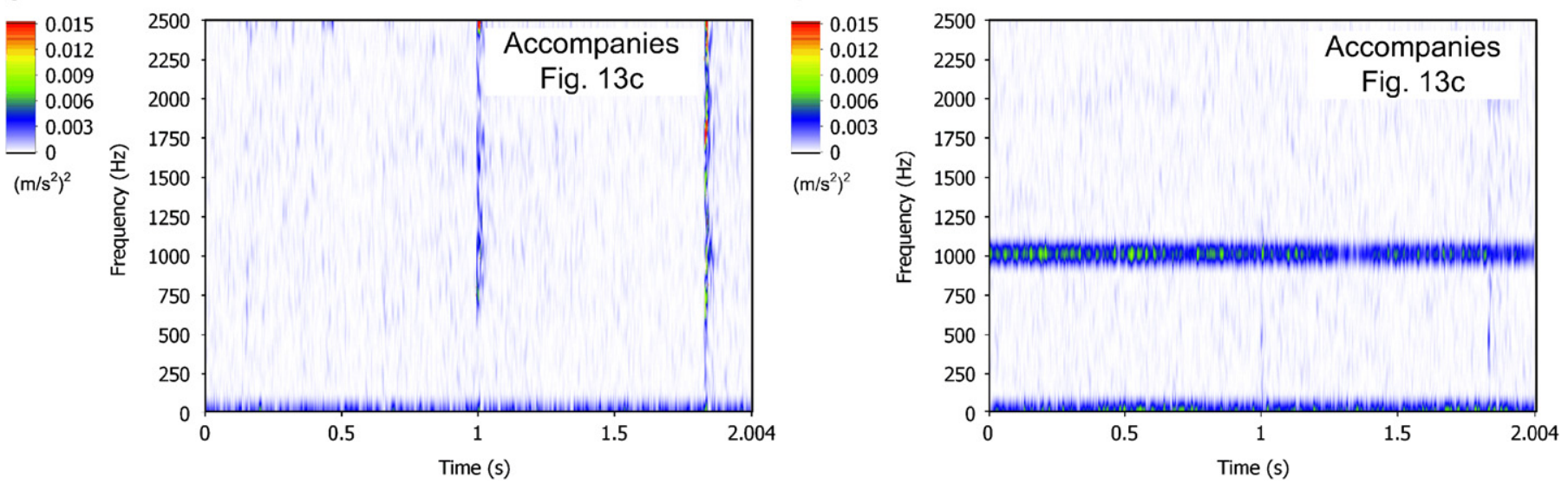

113

114

115

116

117

118

119

120

Fig. 14. Spectrograms of the short-time Fourier transform applied on acceleration data from sensors $\mathrm{CH} 1$ and $\mathrm{CH} 2$. These spectrograms accompany the information obtained from $\mathrm{CHO}(\mathrm{a}-\mathrm{c})$ and indicate that strong $1000 \mathrm{~Hz}$ component was at first captured by sensor $\mathrm{CH} 2$. This means that its source must be close to the inlet flange. Flow conditions are given in Table 1 for data sets grouped under 1.3 (a, b) and for data sets under 1.5 (c, d). 
flange) presented in Fig. 14 accompany information captured by sensor CHO. In particular Fig. 14d reveals that the first appearance of $1000 \mathrm{~Hz}$ resonance frequency was actually captured by sensor $\mathrm{CH} 2$, which is located near the inlet flange. This component was registered at 14:26:41. The intense spectral power of this component abated gradually with time and completely vanished at about 14:31:00 (observed but not presented here). This component was no longer seen neither during the remainder of the first session nor during the second session conducted on 29.01.2009

a

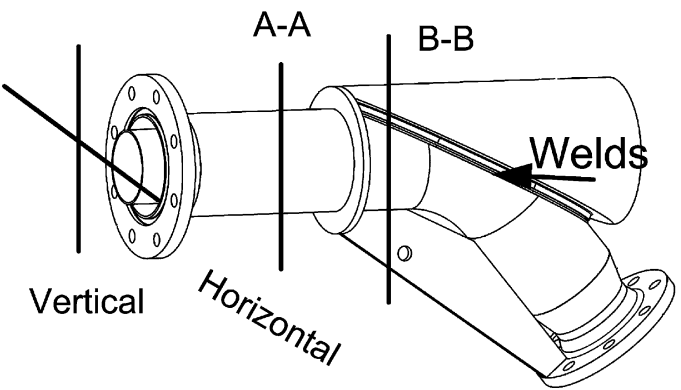

C

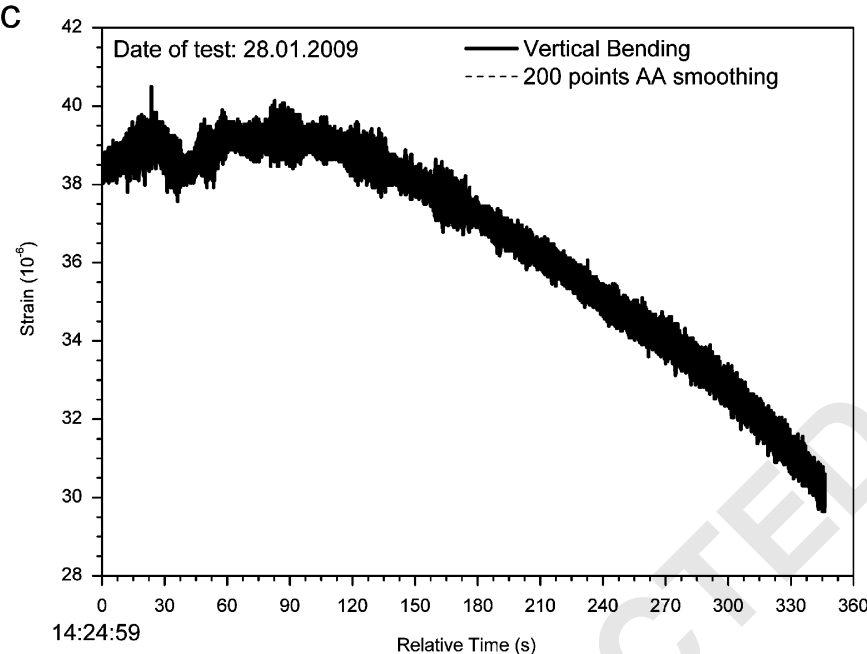

e

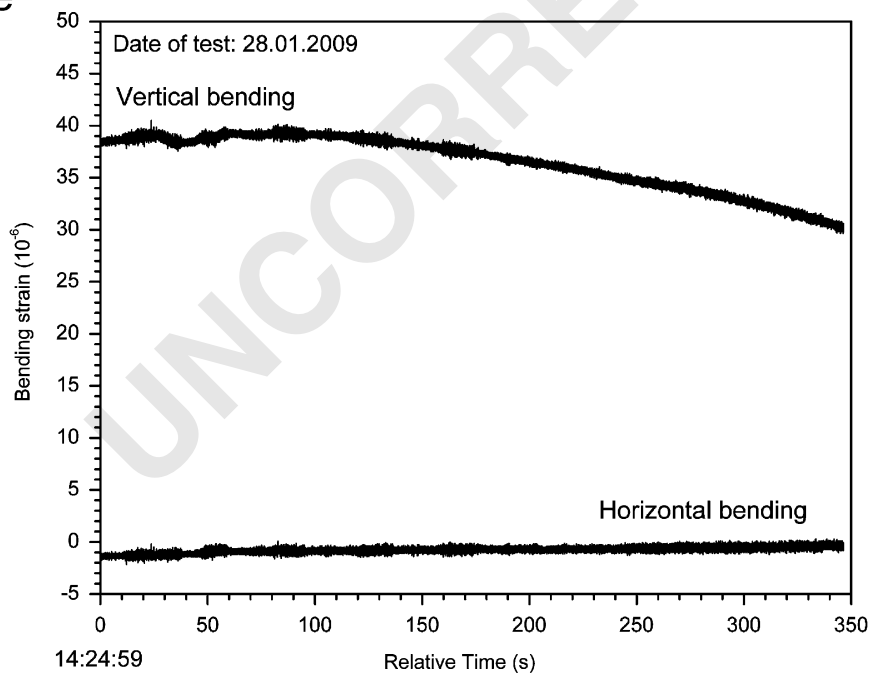

Though such behavior cannot be explained and any discussion about its origin can be speculative, it is very interesting to mention that at about the same time there was a coincidence .Namely, several droplets of mercury were found on the floor below the inlet flange during inspection after the experiment on 28.01.2009 was finalized. Even though it is well-known that a whistling sound can be produced during leakage flows through small cracks or gaps, it cannot be proved or confirmed that a small crack existed and also it cannot be explained from where these droplets came from. The fact is that one of the valves was found faulty and was replaced. d Horizontal Tension

f

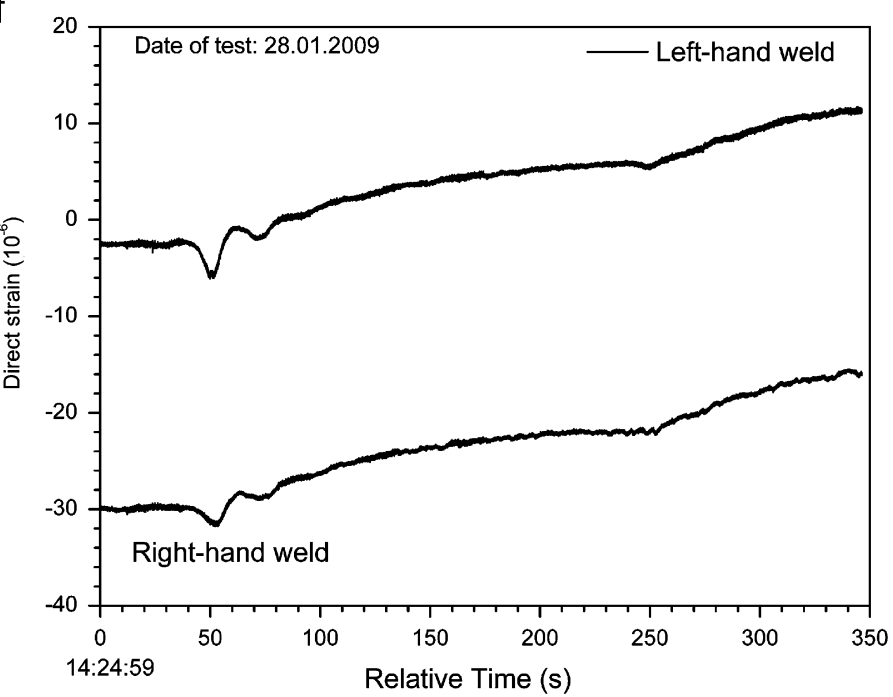
weld strain (f). 
4.2.1.2. Monitoring the test with strain gauges. The level of strain in the hull was monitored by appropriate strain gauges positioned to measure bending across the hull about the vertical and horizontal axes and also at critical positions along the weld of the hull to the support as shown in Fig. 15a and b. The strain can be decomposed in a static component that varies slowly with internal pressure, and a smaller dynamic part superimposed which derives from the interaction of the turbulent liquid metal with the resonance

Fig. 17. LES pressure variations at representative points (top left) are applied to the FEM structural model (top right) by extraction of modal content from FFT processing of the pressure (bottom left, normalized by the average pressure), which, when run in a sine-sweep frequency response analysis, gives displacements on all points of the structural model; the tip of the hull is shown on bottom right. 
modes the fluid excites in the structure. The level of static strain (and hence stress acc. $\sigma=E \varepsilon$ ) varies with the level of pressure exerted on the hull, being itself dependent on the flow rate and static pressure that were varied during the test. The higher the level of pressure, the greater the static bending (in vertical direction) and weld strains (and stresses) due to the bent inlet being subject to forces that tend to rectify the $50^{\circ}$ angle of the bend (Fig. 15b). This matter has been discussed in previous publications on the test [7], and will not be reexamined here. The time at which the strain is graphed corresponds to the flow fluctuation depicted in Fig. 12a and illustrates that the strain gauges are quite sensitive to disturbances in the flow.

On the other hand, the dynamic component of the strain (and stress) is of greater interest to the current discussion. It too contains relevant information pertaining to the response of the structure. In order to filter this dynamic component, a 200-point moving average of the strain signal is subtracted from the same signal, which filters all components below $5 \mathrm{~Hz}$ including the influence of the varying internal pressure and leaves only the significant dynamic component originating from fluid/structure coupling. The proposed method of filtering the strain signal is illustrated in Fig. 15c and d, where the total component of the vertical bending strain is overlaid with the moving average (c), and the resulting subtraction (d) shows all dynamic components above $5 \mathrm{~Hz}$. The dynamic component of the horizontal bending
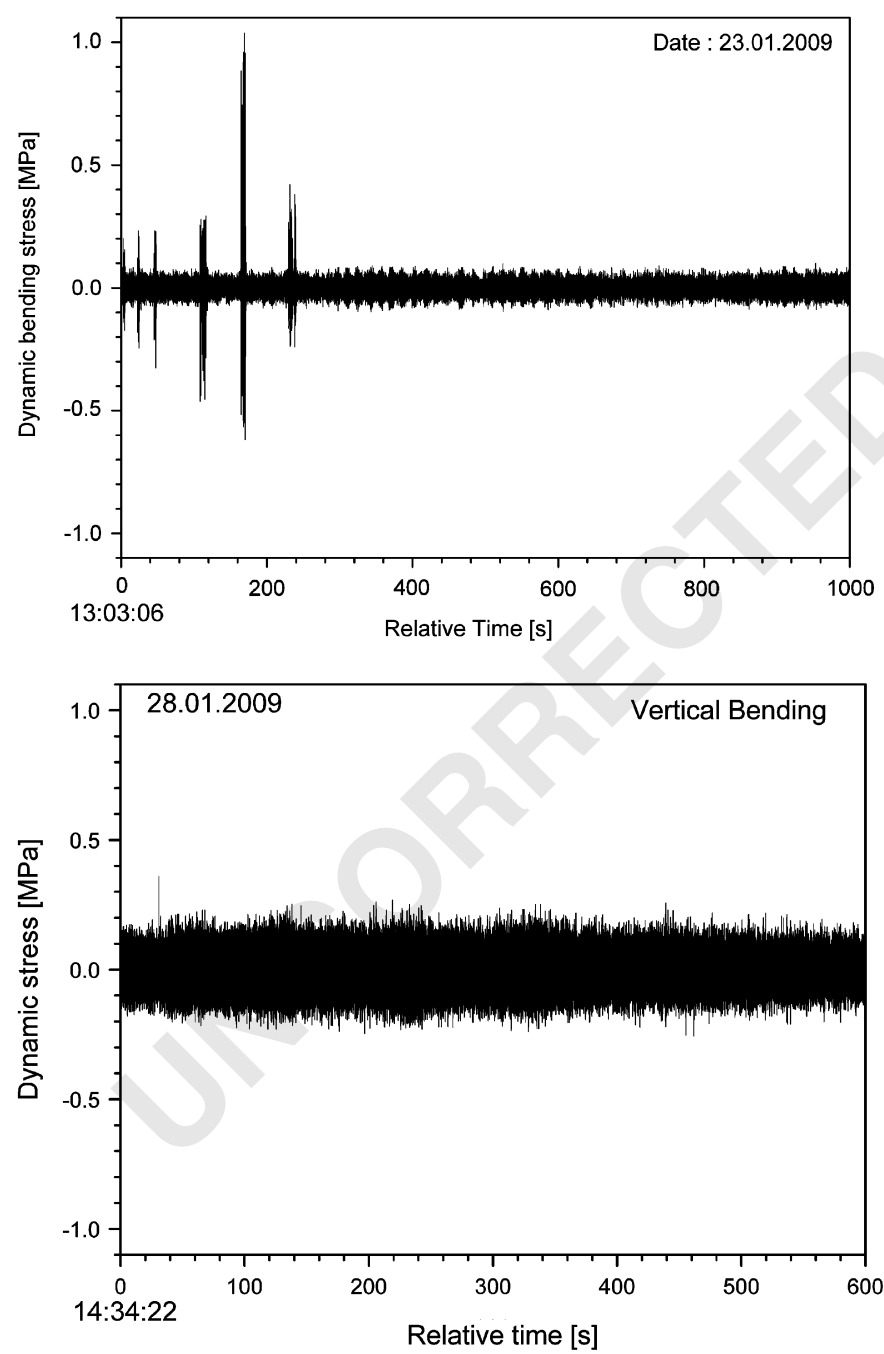

strain is similar to that of the vertical bending strain (e); it differs only as static component of the horizontal strain remains nil and is unaffected by changes in flow or static pressure. This is undoubtedly because of symmetry in the horizontal plane both for the flow and for the structure such that no out-of-balance force can deform the structure as in the vertical plane due to the inlet. The strain gauges positioned along the weld (f) on the other hand are much more affected by internal pressure variations as they respond with greater amplitude than the bending strain gauge pairs to changes in flow or cover gas pressure. The weld strain gauges are relatively sheltered from the dynamic component of the hull vibration as the strain oscillations are lower than on the bending strain gauge pairs, proof that the ribbing welded to the outer hull fulfills its task of reinforcing the hull structure against weld failure.

Another occurrence, referred to as Event 1 in Fig. 7a, which was detected by the accelerometers of the test with flow vanes, was also recorded by the strain gauges, which displayed a tenfold increase in magnitude of the vertical bending stress over a prolonged period, almost a minute (Fig. 16a). This event was followed by a large impact on the structure, which is captured by all strain sensors simultaneously (Fig. 16b) on the 28th at $14: 55: 17.83$. The magnitude of the stress peak exceeded the elastic limit, which was also the upper bound of the data acquisition, resulting in a plateau for three points. The fact that
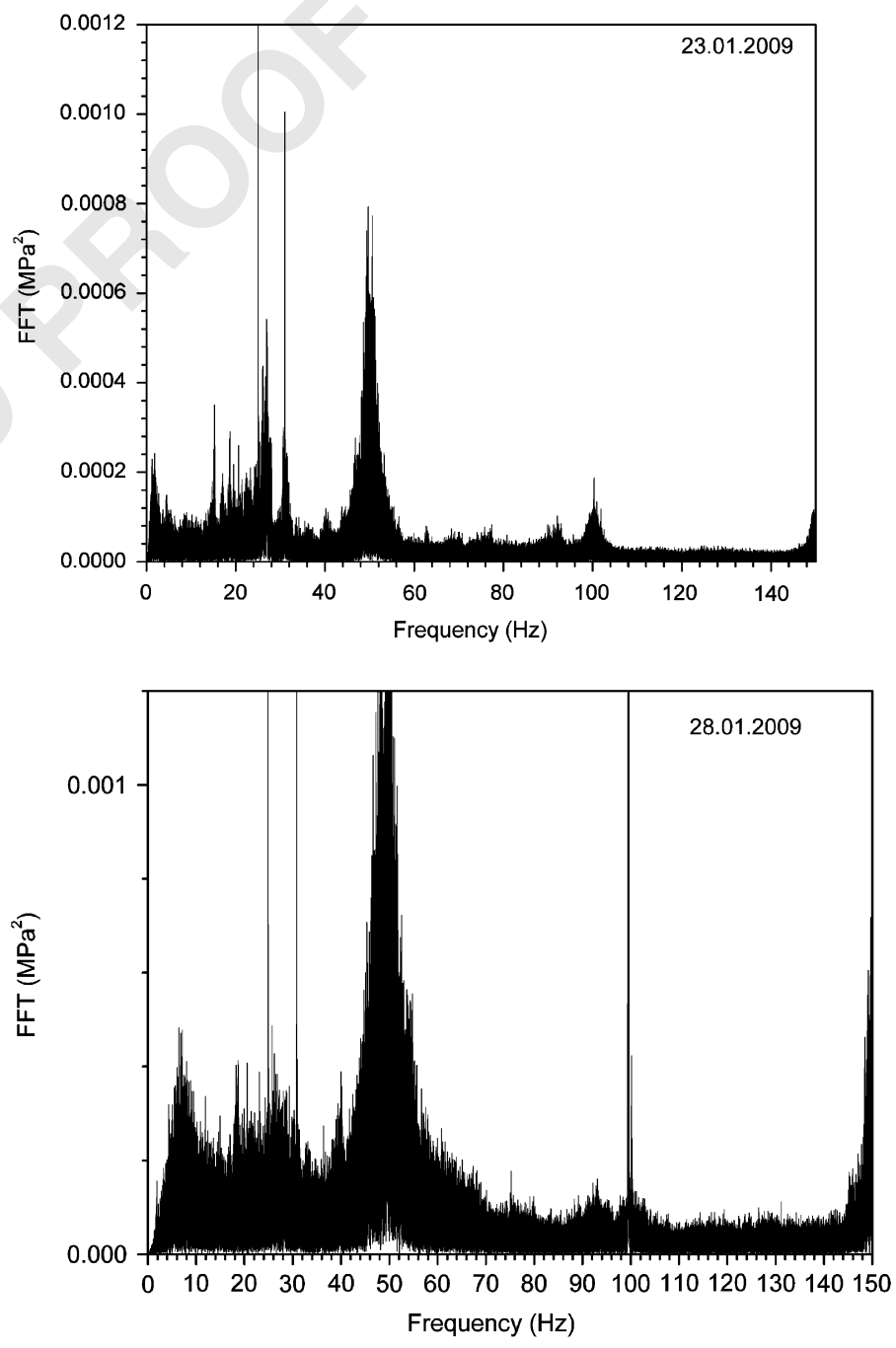

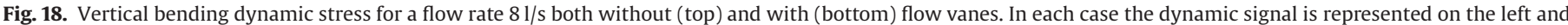
the FFT thereof on the right. 
all strain gauges dropped back exponentially to their original level is an indication that this occurrence was not a measurement error and also that no lasting damage was inflicted to the structure. As mentioned here, above droplets of mercury were found on the floor of the laboratory so it may be that the event recorded in Fig. $16 \mathrm{~b}$ was in some way related to one of the valves, which was later found to be faulty.

The suspected malfunctions were detected half an hour into the test, which were the reasons for the shutdown on the first day, demonstrating how monitoring with multiple redundant sensors can serve to prevent ultimate failure of the facility.

4.2.1.3. Effect of the flow vanes on the overall resonance of the target structure. The FEM calculation was performed on a regular mesh optimised for structural calculations refined in areas of high stress concentration. The structural model shown in Fig. 17 assumed half-symmetry about the vertical plane, an assumption borne out of the fact that major disturbances in the flow arise from the offaxis inlet below the target that imposes disturbances mainly in up or down direction but none horizontally. This assumption was confirmed later in the test where the strain gauge couple monitoring horizontal movement remained at zero until a few minutes into ultimate failure of the flow vanes. The structural model was run in sine sweep mode, in which a range from 10 to $150 \mathrm{~Hz}$ served as the excitation load, using the amplitude of a spatial pressure distribution extracted from the LES calculation. In this manner a one-way coupling was established from the fluid oscillation to the structural dynamic model. This method proved to be relatively accurate in providing orders of magnitude for structural vibrations because there was no feedback mechanism from the structure back into the flow. Such a feedback would have been possible had there been matching oscillations in the flow and the structure. The fact that there were none had been established prior to running the coupled analysis by studying the frequency content of the pressure oscillations derived from the LES and the resonances of the structure under its own weight and that of the fluid.

The realism of this prediction is tested in the figure hereafter, which shows the measured dynamic component. Reproduced on the left of Fig. 18 is the dynamic component of the stress along with the fast Fourier transform (FFT) thereof on the right, for the version of the target without (bottom) and with (top) flow vanes at a flow rate of $8 \mathrm{l} / \mathrm{s}$. The FFT shows a strong resonance between 40 and $50 \mathrm{~Hz}$, which agrees with the prediction shown in Refs.

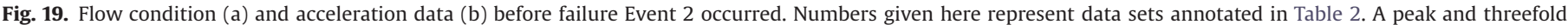
Q2 amplitude increase (b) indicated a failure in the system. 
$1 \quad a$

3

5

0.001

C
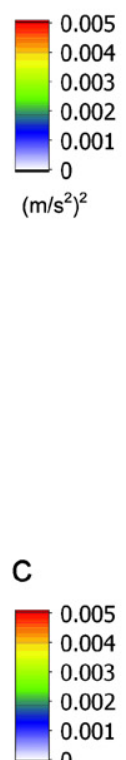

0.005
0.004
0.003
0.002
0.001
0 limits.
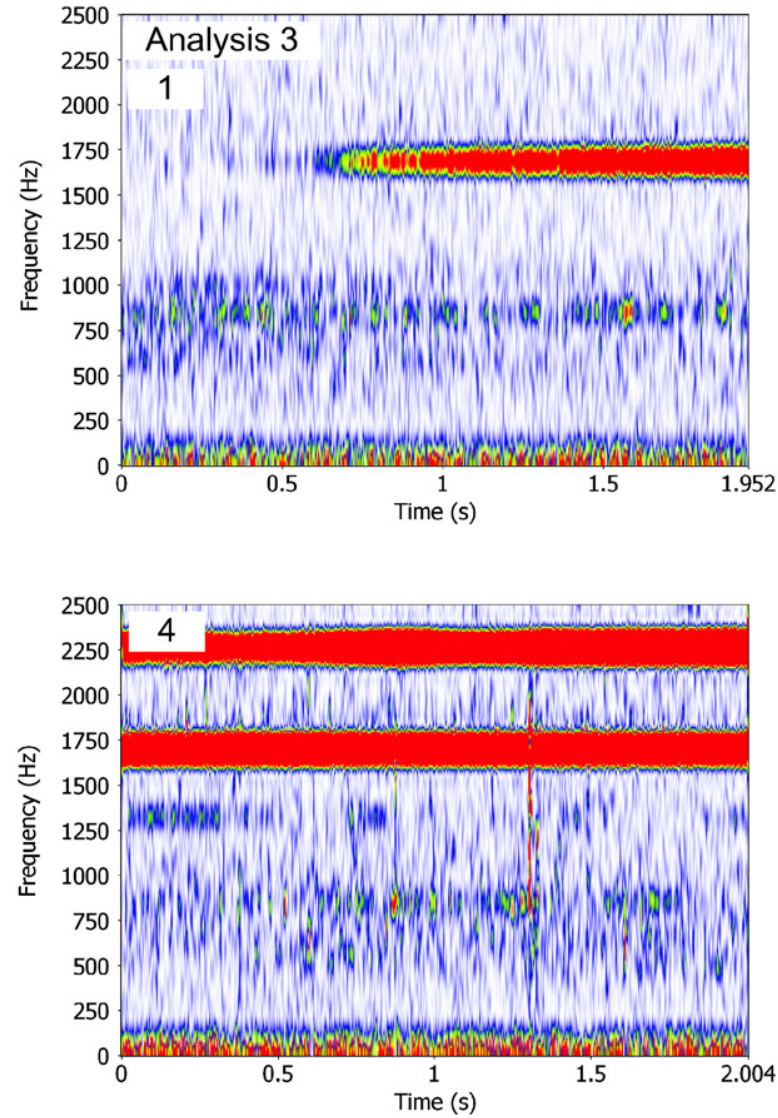

b

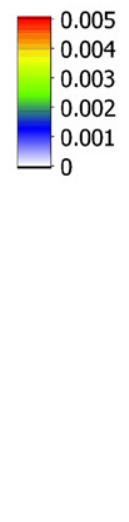

d


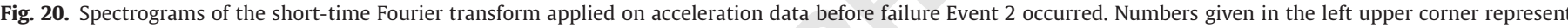

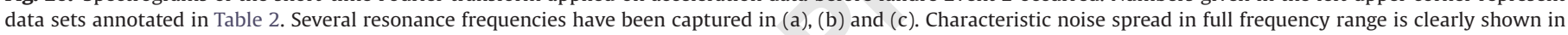
spectrogram (d).

$[5,6]$. The comparison of the two versions shows quite clearly that

1. The magnitude of the dynamic stress had been correctly predicted by the coupled interaction analysis performed with the flow vanes [6] if the results are rationed according to a square law on the flow rate. Indeed the pressure increases with square of the flow rate as do the oscillations in the pressure. Hence a correction factor according to $(12.6 / 8)^{2}=2.56$ may be applied to the measurements. This correction brings in line the measured oscillations $(0.2-0.5 \mathrm{MPa}$ at $8 \mathrm{l} / \mathrm{s})$ and the predicted values ( $1 \mathrm{MPa}$ at $12.6 \mathrm{l} / \mathrm{s})$.

2. The flow vanes increase the vibrations on the hull threefold, but the dynamic component remains well below allowable

3. Thus the validity of the predictions and the small magnitude of the stress at the base of the hull entail that the maximum displacement calculated for the tip of the hull of $50 \mathrm{im}$ is also correct. The target structure is essentially stable both with and without flow vanes.

\subsubsection{The second test (29.01.2009)}

The flow conditions and acceleration data presented in Fig. 19 summarize the cause of events before failure event number 2 . The failure Event 2 happened 15 min before the final failure (Event 3 ) occurred (see Fig. 9). Namely, the pressure in the system was constant and the flow rate was increased from 5 to $7.51 / \mathrm{s}$. The resonance frequencies at about $800,1300,1700$ and $2250 \mathrm{~Hz}$ are captured (Fig. 20). Afterwards, in the second acceleration data set a large peak was detected. The peak had similar characteristics to the peaks in data set 1.2 from 28.01.2009. Twelve seconds after this event the acceleration amplitudes were increased almost threefold, which probably indicates that some spot-welds were broken and a flow vane was detached from its support. Representative spectrograms (sets 1, 2, 4 and 10) of the acceleration signal are shown in Fig. 20. Even though amplitude variations for a constant flow rate and pressure in the system indicate that additional cracks changed the system behavior significantly, spectrogram on Fig. 20d is typical for developed cavitation, when the noise is uniformly spread in full frequency domain. Indeed, the pressure of the cover gas was low (at about 1000 mbar, see Fig. 8a) and cavitation was intensified. The additional load produced by the combined effect of structural resonance and the occurrence of cavitation led to the second failure (Event 2) of the flow vanes. Although the pressure drop (Event 2, Fig. 8a) was increased, the flow rate dropped.

The strain gauge data in Fig. 21 show the final half hour prior to the interruption of the test. Note that in the description hereafter all the measurement segments are consecutive 10 min slots that correspond to the maximum data saving capability of the system operating at very high acquisition rates. There are roughly $1 \mathrm{~min}$ time lapses missing between each of the 10 min segment. The final segment extends to just 2 min before the pump was stopped such that most of the relevant events are covered.

Fig. 21a shows the total stress, comparing the location close to the weld between the hull and support to the bending stress in the vertical plane (refer also to Fig. 15 for positions). This serves to 


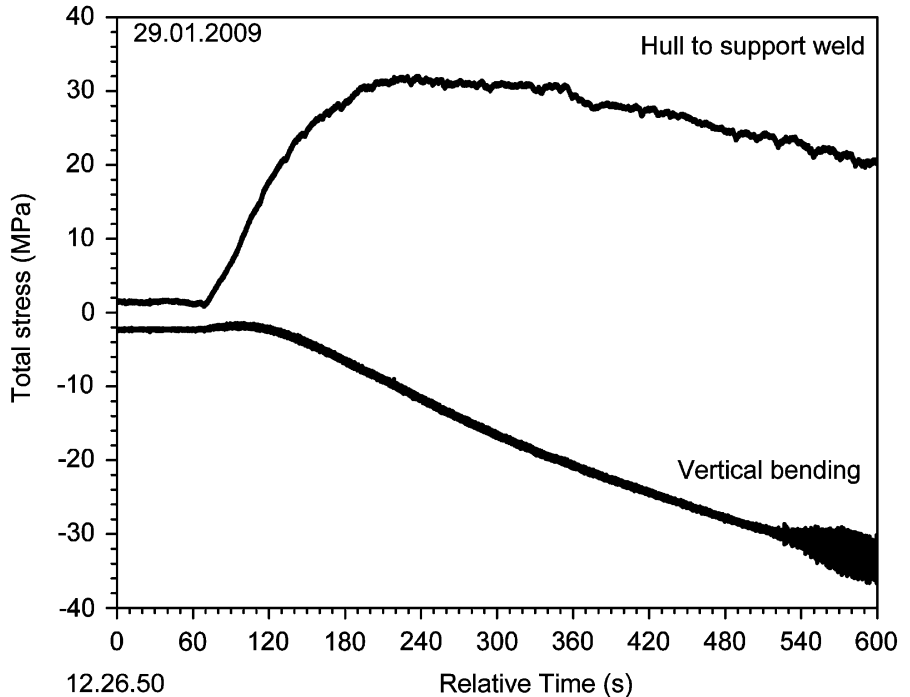

C

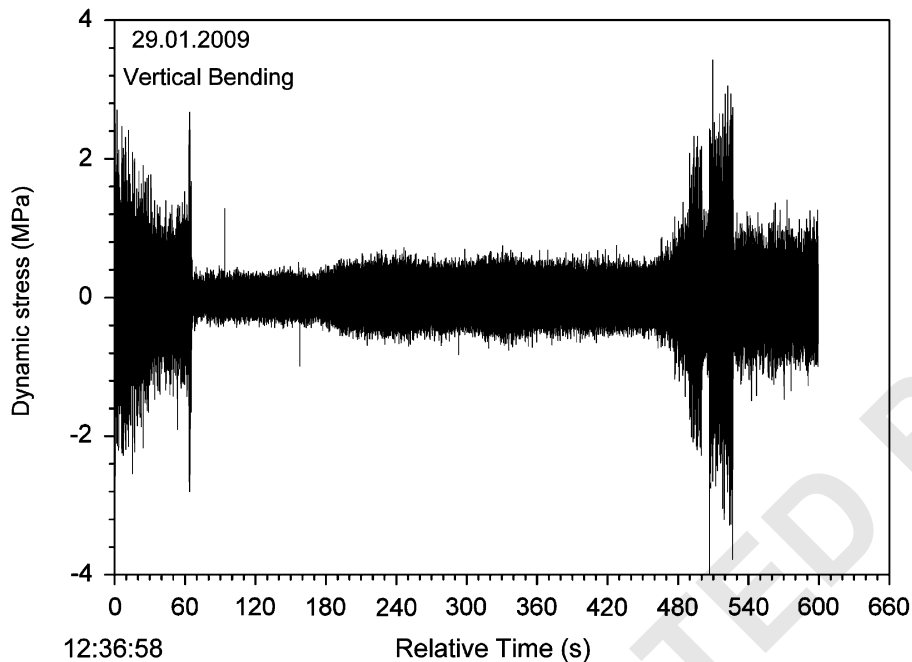

b

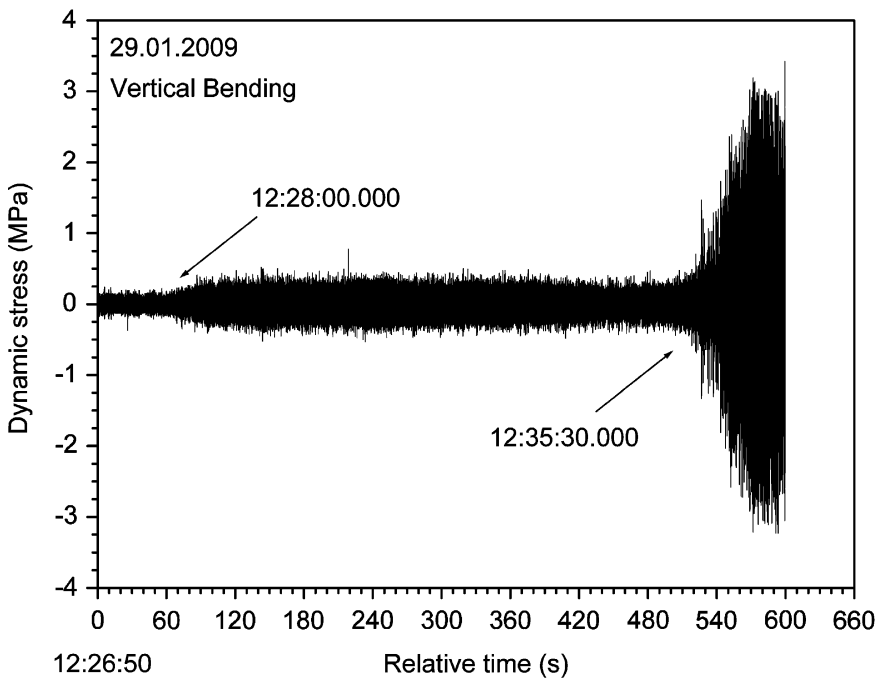

d

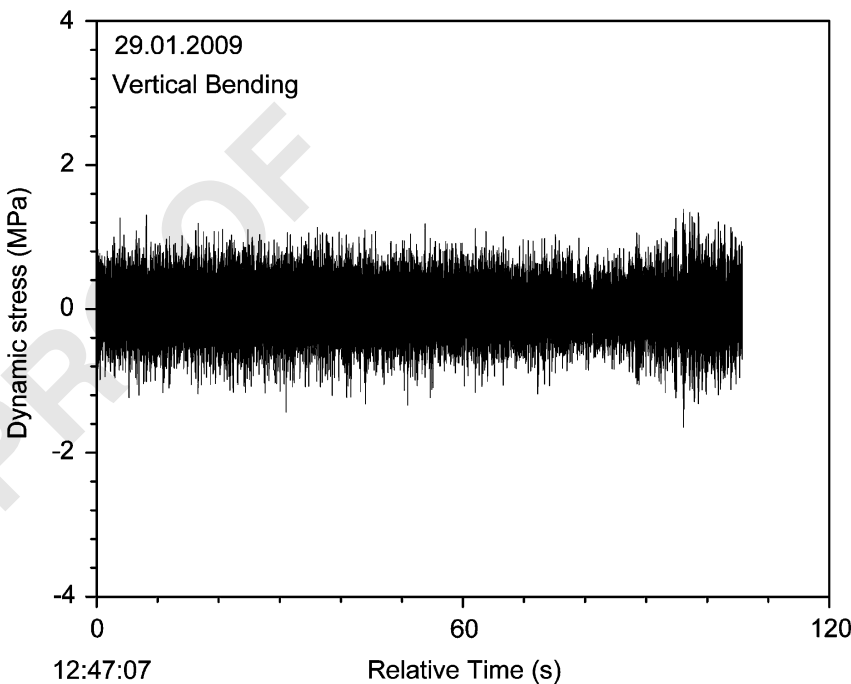

Fig. 21. Vertical bending dynamic stress prior to ultimate failure of one of the flow vanes.

illustrate the sudden onset of increased bending oscillations with the rupture process. The hull-to-support weld remains unaffected by the oscillations, proof that the reinforcements added to the structure of the hull are performing well in isolating the consequences of the internal rupture.

Fig. 21b and those thereafter show the dynamic component of the vertical bending stress. The evolution in time confirms the observation made above that the process of rupture of the flow vane weld onto the guide tube was gradual, probably originating on one of the spot-welds, which led to an overload on the other spot-welds, which in turn gave way to a gradual "unzipping" of all the spot-welded connections of the flow vane attached to the guide tube.

The initial start of the rupture (Fig. 21c) is clearly marked by an increase in dynamic stress from $1 / 4$ [MPa] to over $1 / 2$ [MPa], in line with the observation made at the same time by the accelerometers in Fig. 19b. Finally just minutes before final interruption of the test, there is a tenfold increase in the magnitude of the stress at 12:35:30.00, which corresponds to "Event 2" marked by a sudden increase in pressure loss. We can reconstruct that this phase corresponds to the complete detachment of the flow vane.

Once the flow vane has detached, the dynamic bending stress on the hull (Fig. 21d) shows alternating patterns of high bending followed by temporary reduction in magnitude, probably depending on how the detached flow vanes are jammed in the internal structure around the window. There is however no further increase in oscillations, indicating that the remainder of the flow vanes closer to the window remain attached and do not add to the oscillations of the structure. This is confirmed when the target is opened and the damage inspected.

After the final failure event (Event 3, Fig. 9) occurred at $12: 45: 17.777$, the experiment was stopped and all recorded data successfully were saved.

\section{Conclusions}

The EURISOL initiative gave the PSI an opportunity to propose an innovative concept for the most powerful neutron converter target ever built: a design that incorporated advanced features 
such as flow vanes for reversing and stabilising the fluid over the beam window.

The flow vanes and the cusp window were an unproven concept that had never been tested with liquid metal. CFD and FEM calculations showed that such a design would lead to a lowpressure drop and optimal cooling conditions along the beam window. Unfortunately, the analysis had also showed that the flow vanes, as currently designed, would probably break because of fatigue at some undetermined time during the test. Having forewarning and by taking adequate safety precautions, the team was able to proceed with the test and collect data until the point of rupture. This allowed sufficient data to be gathered on the flow vanes; indeed, they survived long enough to be able to check their performance against predictions. The comparison was satisfactory and gives the team confidence the design may be improved as they are necessary to stabilise a liquid metal flow over a highly heated beam window surface.

A second achievement, in many ways as important, was to prove that it is possible to use remote monitoring techniques such as an external microphone, accelerometers or strain gauges to monitor accurately the health of a liquid metal target, including internal components, which are blanketed from standard measurement techniques because of the opacity and density of the liquid metal. This major proven capability will improve protection, safeguard and shutdown procedures on facilities using highpower target under irradiation in the future.

\section{Uncited references}

\section{Acknowledgments}

The authors are grateful to August Kalt, Sergej Ivanov, Ernests Platacis and Anatolij Zik for their valuable assistance, numerous technical contributions to the design and erection of the experimental installation and for carrying out the experiments.

Valuable contributions to the test set-up, in particular to data acquisition, by Enzo Manfrin are gratefully acknowledged. Fillipo Barbagallo provided very useful ancillary equipment for placing thermocouples at the decisive locations.

The authors are thankful to Knud Thomsen for fruitful discussions, advice and support.

We acknowledge the financial support of the European Community under the FP6 "Research Infrastructure ActionStructuring the European Research Area" EURISOL DS Project Contract no. 515768 RIDS. The EC is not liable for any use that can be made on the information contained herein.

\section{References}

[1] ANSYS Inc., 2007. ANSYS CFX Solver Modelling Guide, CFX 11.0, 2007.

[2] C.E. Brennen, Cavitation and Bubble Dynamics, Oxford University Press, Oxford, 1995.

[3] Internet source, 2009. 〈http://www.eurisol.org >, 2009.

[4] K. Samec, Design of the EURISOL converter target, PSI Internal Note 34-07-05, 2007.

[5] K. Samec, R. Milenkovic, D. Demetjevs, M. Ashrafi-Nik, A. Kalt, Nucl. Instr. and Meth. A xx (2009) xx.

[6] K. Samec, et al., Eurisol-DS multi-MW target fluid-structure interaction, CERN note no. CERN-EN-Note-2009-005 STI, 2009.

[7] R. Milenkovic, D. Sergejs, K. Samec, E. Platacis, A. Zik, A. Flerov, K. Thomsen, Nucl. Instr. and Meth. A xx (2009) xx

[8] S. Mallat 1999. A wavelet tour of signal processing ISBN: 978-0-12-466606-1.

[9] A. Paidoussis, J. Fluids Struct. 22 (2006) 741-755.

[10] W. Wagner, F. Groeschel, K. Thomsen, H. Heyck, J. Nucl. Mater. 377 (2008) $12-16$. 